\title{
Gauge-invariant Green's functions for the bosonic sector of the standard model
}

\author{
Andreas Nyffeler* \\ DESY, Platanenallee 6, D-15738 Zeuthen, Germany \\ Andreas Schenk \\ Hambacher Straße 14, D-64625 Bensheim-Gronau, Germany
}

(19 June 2000)

\begin{abstract}
There are many applications in gauge theories where the usually employed framework involving gauge-dependent Green's functions leads to considerable problems. In order to overcome the difficulties invariably tied to gauge dependence, we present a manifestly gauge-invariant approach. We propose a generating functional of appropriately chosen gauge-invariant Green's functions for the bosonic sector of the standard model. Since the corresponding external sources emit one-particle states, these functions yield the same $S$-matrix elements as those obtained in the usual framework. We evaluate the generating functional for the bosonic sector of the standard model up to the one-loop level and carry out its renormalization in the on-shell scheme. Explicit results for some two-point functions are given. Gauge invariance is manifest at any step of our calculation.

PACS number(s): 11.15.Bt, 11.15.Ex, 12.15.-y
\end{abstract}

\section{INTRODUCTION}

The concept of gauge symmetry has played an important role in the development of quantum field theory and particle physics. Nevertheless, most practical calculations in perturbation theory are done by fixing a gauge at the beginning and using gauge-dependent Green's functions [1.22. Gauge dependence manifests itself only in the off-shell behavior of these Green's functions. Their pole positions and residues, i.e., particle masses, decay widths, and $S$ matrix elements, are gauge-independent. As long as one is only dealing with physical, and therefore, gauge-invariant quantities there seems to be no reason why the gauge symmetry should be manifest throughout the whole calculation.

There are, however, situations where one is interested to gain information from off-shell quantities or where one is forced to deal with them. For instance, problems with the gauge-dependent approach arise when one is dealing with finite width effects of unstable particles, which is relevant for $W$-boson production at the CERN $e^{+} e^{-}$collider LEP2 or at future colliders [3]. Another example are the oblique parameters $S, T, U$, [4] which parametrize effects of new physics on the vacuum polarization of the electroweak gauge bosons and which are defined through gaugedependent self-energies. Off-shell information [5] is also used to investigate the electroweak phase transition where one is studying the effective potential at finite temperature. In the context of effective field theories one encounters gauge-dependencies [6], if one includes electromagnetic effects [7] in chiral perturbation theory [8]. Another example where a gauge-dependent framework causes considerable complications is the matching of a full and an effective theory. As pointed out in Refs. [9, 10], there are some subtleties involved concerning gauge invariance, if the matching is performed at the level of gauge-dependent Green's functions.

Several attempts have been made in order to solve these problems with gauge-dependencies in different applications. For instance, the fermion loop scheme [11] was developed to treat unstable particles in $W$-pair production. The pinchtechnique [12,13] was used to define quantities $S, T, U$ which are independent of the gauge-parameter 14]. Another approach to improve the properties of Green's functions with respect to gauge transformations is the background field method [15] which was applied to the standard model in Ref. [16].

The techniques employed differ in the degree with which the symmetry properties are manifest. All these approaches work within the usually employed gauge-fixed framework and try to improve the properties of Green's functions with respect to the gauge symmetry. Instead, we proposed a manifestly gauge-invariant functional approach in Ref. [10] which is better suited for the applications we have in mind. It deals from the beginning only with Green's functions of gauge-invariant operators. We first applied our method to an effective field theory analysis of the Abelian Higgs model. Later we showed how one can treat charged particles with this new method in a manifestly gauge-invariant

\footnotetext{
*Present address: Centre de Physique Théorique, CNRS-Luminy, Case 907, F-13288 Marseille Cedex 9, France; Email address: nyffeler@cpt.univ-mrs.fr
} 
way [17]. This was done by a detailed comparison of QED in our approach with the conventional, gauge-dependent method. The extension to the electroweak standard model was briefly sketched in that reference as well.

In the present paper we discuss the application of our method to the non-Abelian case in full detail. Generalizing the functional methods developed in Refs. 18, 10,17] we construct a generating functional for appropriately chosen gauge-invariant Green's functions for the bosonic sector of the standard model at the one-loop level. This is done by coupling external sources to gauge-invariant operators in such a way that the sources emit one-particle states of the Higgs boson, the $W$ - and $Z$-boson and the photon. Due to the manifestly preserved gauge symmetry in our approach, the generating functional and the corresponding Green's functions automatically exhibit useful physical properties. In the usual approach to gauge theories these properties have to be imposed by specific renormalization conditions or by employing Ward identities. Finally, we show how one can extract physical quantities like masses, coupling constants and $S$-matrix elements from these gauge-invariant Green's functions. Since the external sources emit one-particle states, the gauge-invariant Green's functions lead to the same $S$-matrix elements as those obtained in the usual framework. In a first step we have not included fermions in our analysis. In principle, the treatment of fermions is straightforward in our approach. The corresponding source terms have been written down already in Ref. [17].

Because we couple sources only to gauge-invariant operators it is possible to calculate the generating functional and the corresponding Green's functions without fixing a gauge in the path integral [10,17]. At tree level we can solve the equations of motion for the physical degrees of freedom and define their propagators. The manifestly gauge-invariant method generalizes to the one-loop level where no Faddeev-Popov ghost fields appear. We note that the propagators which enter loop diagrams are not identical to the ones in unitary gauge in the usual framework. Therefore, the Green's functions of our gauge-invariant operators have a decent high-energy behavior and the renormalizability of the theory is clearly visible. This is due to the fact that the Goldstone boson modes are still present in the loops. We will discuss the renormalization of the theory in the presence of external sources in detail, using dimensional regularization and employing heat-kernel techniques.

In the present work we concentrate on the development of a gauge-invariant functional approach to the symmetry breaking sector of the standard model in the spontaneously broken phase, thereby laying the theoretical foundations. A first application of our gauge-invariant method can be found in Ref. [19] where we have performed a detailed analysis of the electroweak chiral Lagrangian 20, which describes the low-energy structure of a strongly interacting electroweak symmetry breaking sector. In Ref. 19] we have studied two issues related to gauge invariance where the usual approach with gauge-dependent Green's functions leads to considerable problems. In particular, we have determined the number of independent parameters in the effective Lagrangian by making use of the equations of motion to remove redundant terms. Furthermore, we have evaluated the effective Lagrangian for the standard model with a heavy Higgs boson. The calculation was performed by matching gauge-invariant Green's functions in the full and the effective theory at low energies.

Finally, we would like to stress that the construction of the generating functional is done in such a way that the gauge symmetry is manifestly preserved at any stage. In this respect our method differs from the treatment of charged particles as proposed in Refs. [21,22], although the starting point for the choice of gauge-invariant fields is very similar. We note that there are other attempts in the literature to define gauge-invariant and gauge-independent Green's functions in field theories, for instance the Vilkovisky-DeWitt effective action [23]. Another method which naturally deals with gauge-invariant objects is of course lattice gauge theory [24].

This paper is organized as follows: In the next section we discuss our choice of gauge-invariant operators and the corresponding external sources which emit one-particle states of the bosons. Then we define the generating functional for the Green's functions. In Sec. III we evaluate the generating functional at tree level. In particular, we discuss the solutions of the equations of motion for the physical degrees of freedom. In Sec. IV we calculate the generating functional at the one-loop level in such a way that the gauge symmetry is manifestly preserved throughout. The result encodes all one loop effects of the theory. In Sec. V we discuss the renormalization of the model and determine the renormalization prescriptions. In Sec. VI] we calculate the two-point functions for the gauge-invariant operators and present some properties of these Green's functions which follow from the gauge symmetry. Furthermore we extract the electric charge and the masses of the bosons from the relevant two-point functions. Finally, we summarize our results in Sec. VII. Some technical details and lengthy expressions which are needed for the calculation can be found in several Appendices.

\footnotetext{
${ }^{1}$ Note that we distinguish between the Green's functions, like two-point or $n$-point functions, of gauge-invariant operators which are obtained from the generating functional and the propagators which appear in the solutions of the equations of motion at tree level or within loop integrals.
} 


\section{THE LAGRANGIAN AND THE GAUGE-INVARIANT GENERATING FUNCTIONAL}

The Lagrangian of the standard model without fermions is of the form

$$
\mathcal{L}=\frac{1}{2} D_{\mu} \Phi^{\dagger} D_{\mu} \Phi-\frac{1}{2} m^{2} \Phi^{\dagger} \Phi+\frac{\lambda}{4}\left(\Phi^{\dagger} \Phi\right)^{2}+\frac{1}{4 g^{2}} W_{\mu \nu}^{a} W_{\mu \nu}^{a}+\frac{1}{4 g^{\prime 2}} B_{\mu \nu} B_{\mu \nu},
$$

where $\Phi=\left(\begin{array}{c}\phi^{1} \\ \phi^{2}\end{array}\right)$ denotes the Higgs boson doublet which is coupled to the $S U(2)_{L}$ gauge fields $W_{\mu}^{a}(a=1,2,3)$ and the $U(1)_{Y}$ gauge field $B_{\mu}$ through the covariant derivative

$$
D_{\mu} \Phi=\left(\partial_{\mu}-i \frac{\tau^{a}}{2} W_{\mu}^{a}-i \frac{1}{2} B_{\mu}\right) \Phi .
$$

Note that we have absorbed the coupling constants $g$ and $g^{\prime}$ into the gauge fields $W_{\mu}^{a}$ and $B_{\mu}$, respectively. The field strengths are given by

$$
\begin{aligned}
W_{\mu \nu}^{a} & =\partial_{\mu} W_{\nu}^{a}-\partial_{\nu} W_{\mu}^{a}+\varepsilon^{a b c} W_{\mu}^{b} W_{\nu}^{c}, \\
B_{\mu \nu} & =\partial_{\mu} B_{\nu}-\partial_{\nu} B_{\mu} .
\end{aligned}
$$

The Higgs field $\Phi$ and the gauge fields $W_{\mu}^{a}, B_{\mu}$ transform under $S U(2)_{L}$ gauge transformations in the following way:

$$
\begin{aligned}
\Phi & \rightarrow \mathcal{V} \Phi, \quad \mathcal{V} \in S U(2), \\
W_{\mu} & \rightarrow \mathcal{V} W_{\mu} \mathcal{V}^{\dagger}-i\left(\partial_{\mu} \mathcal{V}\right) \mathcal{V}^{\dagger}, \quad W_{\mu} \equiv W_{\mu}^{a} \frac{\tau^{a}}{2}
\end{aligned}
$$

and under $U(1)_{Y}$ gauge transformations as follows:

$$
\begin{aligned}
\Phi & \rightarrow e^{-i \omega / 2} \Phi, \\
B_{\mu} & \rightarrow B_{\mu}-\partial_{\mu} \omega .
\end{aligned}
$$

For computational convenience we are working in Euclidean space-time.

For $m^{2}>0$ the classical potential has its minimum at a nonzero value $\Phi^{\dagger} \Phi=m^{2} / \lambda$ and the $S U(2)_{L} \times U(1)_{Y}$ symmetry is spontaneously broken down to $U(1)_{\mathrm{em}}$. Accordingly, the field $\Phi$ describes one massive mode, the Higgs particle, and three Goldstone bosons which render the gauge fields $W$ and $Z$ massive. Finally, the spectrum contains the massless photon. At tree level, the masses and the electric coupling constant $e$ are given by the relations

$$
M_{H}^{2}=2 m^{2}, M_{W}^{2}=\frac{m^{2} g^{2}}{4 \lambda}, M_{Z}^{2}=\frac{m^{2}\left(g^{2}+g^{\prime 2}\right)}{4 \lambda}, e^{2}=\frac{g^{2} g^{2}}{g^{2}+g^{\prime 2}} .
$$

Furthermore we will use the following definition of the weak mixing angle:

$$
c^{2} \equiv \cos ^{2} \theta_{W}=M_{W}^{2} / M_{Z}^{2}, s^{2} \equiv 1-c^{2} .
$$

In order to have nontrivial solutions of the equations of motion, we furthermore couple external sources to the gauge fields and the Higgs boson. As discussed in detail for the Abelian Higgs model in Ref. [10] and for QED in Ref. [17], the appropriate choice of the source terms is crucial for a manifestly gauge-invariant analysis.

The sources will only respect the gauge symmetry, if they do not couple to the gauge degrees of freedom. Otherwise, one has to impose constraints on the fields in order to solve the equations of motion. Usually, this problem is cured by fixing a gauge. However, one can also turn the argument around and consider only those external sources which couple to gauge-invariant operators. As we will see below, such a manifestly gauge-invariant treatment is in fact possible at the classical level as well as when quantum corrections are taken into account.

In order to write down appropriate source terms we will introduce another set of fields for the dynamical degrees of freedom which are already invariant under the non-Abelian group $S U(2)_{L}$ and, in parts, under the Abelian group $U(1)_{Y}$ as well. It has been known for a long time 25 27] that all fields in the standard model Lagrangian can be written, in the spontaneously broken phase, in a gauge-invariant way up to the unbroken $U(1)_{\mathrm{em}}$. It is convenient to use a polar representation for the Higgs doublet field

$$
\Phi=\frac{m}{\sqrt{\lambda}} R U
$$


where the unitary field $U$, satisfying $U^{\dagger} U=1$, describes the three Goldstone bosons, while the radial component $R$ represents the Higgs boson. Furthermore, we define the $Y$-charge conjugate doublet

$$
\widetilde{\Phi}=i \tau_{2} \Phi^{*},
$$

and similarly, $\widetilde{U}=i \tau_{2} U^{*}$.

We introduce the following operators:

$$
\begin{aligned}
V_{\mu}^{1} & =i \widetilde{\Phi}^{\dagger} D_{\mu} \Phi+i \Phi^{\dagger} D_{\mu} \widetilde{\Phi}=\frac{m^{2}}{\lambda} R^{2} \mathcal{W}_{\mu}^{1}, \\
V_{\mu}^{2} & =-\widetilde{\Phi}^{\dagger} D_{\mu} \Phi+\Phi^{\dagger} D_{\mu} \widetilde{\Phi}=\frac{m^{2}}{\lambda} R^{2} \mathcal{W}_{\mu}^{2}, \\
V_{\mu}^{3} & =i \widetilde{\Phi}^{\dagger} D_{\mu} \widetilde{\Phi}-i \Phi^{\dagger} D_{\mu} \Phi=\frac{m^{2}}{\lambda} R^{2} \mathcal{Z}_{\mu},
\end{aligned}
$$

involving the gauge boson fields

$$
\begin{aligned}
\mathcal{W}_{\mu}^{+} & =\frac{i}{2}\left(\tilde{U}^{\dagger}\left(D_{\mu} U\right)-\left(D_{\mu} \tilde{U}\right)^{\dagger} U\right), \\
\mathcal{W}_{\mu}^{-} & =\frac{i}{2}\left(U^{\dagger}\left(D_{\mu} \tilde{U}\right)-\left(D_{\mu} U\right)^{\dagger} \tilde{U}\right), \\
\mathcal{Z}_{\mu} & =i\left(\tilde{U}^{\dagger}\left(D_{\mu} \tilde{U}\right)-U^{\dagger}\left(D_{\mu} U\right)\right), \\
\mathcal{A}_{\mu} & =B_{\mu}+s^{2} \mathcal{Z}_{\mu}, \\
\mathcal{W}_{\mu}^{ \pm} & =\frac{1}{2}\left(\mathcal{W}_{\mu}^{1} \mp i \mathcal{W}_{\mu}^{2}\right),
\end{aligned}
$$

which are invariant under the $S U(2)_{L}$ gauge transformations from Eq. (2.5). Up to a constant factor the operators $V_{\mu}^{i}$ in Eq. (2.11) correspond to the currents of the global symmetry $S U(2)_{R}$.

In terms of these composite fields the Lagrangian from Eq. (2.1) reads

$$
\mathcal{L}_{\mathrm{SM}}^{0}=\frac{1}{2} \frac{m^{2}}{\lambda}\left[\partial_{\mu} R \partial_{\mu} R-m^{2} R^{2}+\frac{m^{2}}{2} R^{4}+R^{2}\left(\mathcal{W}_{\mu}^{+} \mathcal{W}_{\mu}^{-}+\frac{1}{4} \mathcal{Z}_{\mu} \mathcal{Z}_{\mu}\right)\right]+\frac{1}{4 g^{2}} \mathcal{W}_{\mu \nu}^{a} \mathcal{W}_{\mu \nu}^{a}+\frac{1}{4{g^{\prime}}^{2}} B_{\mu \nu} B_{\mu \nu}
$$

where

$$
\begin{aligned}
\mathcal{W}_{\mu \nu}^{a} & =\partial_{\mu} \mathcal{W}_{\nu}^{a}-\partial_{\nu} \mathcal{W}_{\mu}^{a}+\varepsilon^{a b c} \mathcal{W}_{\mu}^{b} \mathcal{W}_{\nu}^{c}, a=1,2,3, \\
\mathcal{W}_{\mu}^{3} & =\mathcal{Z}_{\mu}+B_{\mu} .
\end{aligned}
$$

In order to calculate Green's functions from which we then can extract physical quantities like masses, coupling constants and $S$-matrix elements, we have to introduce external sources which emit one-particle states of the Higgs field and the gauge bosons. In analogy to the Abelian case [10,17] we couple sources to the $S U(2)_{L} \times U(1)_{Y}$ gaugeinvariant operator $\Phi^{\dagger} \Phi$ and the field strength $B_{\mu \nu}$. For the massive gauge bosons the situation is more involved. Whereas the field $\mathcal{Z}_{\mu}$ is fully gauge-invariant, the charged gauge fields $\mathcal{W}_{\mu}^{ \pm}$and the corresponding currents $V_{\mu}^{ \pm}$have a residual gauge dependence under the $U(1)_{Y}$ gauge transformations from Eq. (2.6):

$$
\mathcal{W}_{\mu}^{ \pm} \rightarrow e^{\mp i \omega} \mathcal{W}_{\mu}^{ \pm} \quad, \quad V_{\mu}^{ \pm} \rightarrow e^{\mp i \omega} V_{\mu}^{ \pm}
$$

We can, however, compensate this gauge dependence by multiplying the charged fields $\mathcal{W}_{\mu}^{ \pm}$and $V_{\mu}^{ \pm}$by a phase factor [21,22, 17]. In terms of the operators $V_{\mu}^{a}$ from Eq. 2.11) we can then write appropriate $S U(2)_{L} \times U(1)_{Y}$ gauge-invariant source terms for all the fields as follows:

$$
\mathcal{L}_{\text {source }}^{1}=-\frac{1}{2} h \Phi^{\dagger} \Phi-\frac{1}{2} K_{\mu \nu} B_{\mu \nu}+J_{\mu}^{a} \varphi^{a b} V_{\mu}^{b},
$$

with external sources $h, K_{\mu \nu}$, and $J_{\mu}^{a}(a=1,2,3)$. The phase factor in Eq. (2.21) is defined by

$$
\varphi(x)=\exp \left(T \int d^{d} y \mathcal{G}_{0}(x-y) \partial_{\mu} B_{\mu}(y)\right),
$$


with

$$
T=\left(\begin{array}{ccc}
0 & 1 & 0 \\
-1 & 0 & 0 \\
0 & 0 & 0
\end{array}\right)
$$

and

$$
\mathcal{G}_{0}(x-y)=\left\langle x\left|\frac{1}{-\square}\right| y\right\rangle .
$$

Since the vacuum in the spontaneously broken phase corresponds to the value $R=1$, Green's functions of the field $\Phi^{\dagger} \Phi$ contain one-particle poles of the Higgs boson, whereas those of $\varphi^{a b} V_{\mu}^{b}$ have one-particle poles of the gauge bosons $W$ and $Z$.

In Ref. [17] it was shown to all orders in perturbation theory that a phase factor $\varphi$ which is defined analogously to Eq. (2.22) does not spoil the renormalizability of QED. Since the proof did not rely on any particular feature of QED, the same should be true for the present case as well. This is due to the fact that the phase factor only contains the Abelian gauge degree of freedom which does not affect the dynamics of the theory. Since the operator $\Phi^{\dagger} \Phi$ and the currents $V_{\mu}^{a}$ from Eq. (2.11) have dimension less than four, source terms involving these operators do not spoil the renormalizability either. The reader should note, however, that we do not have a formal proof of renormalizability to all orders in perturbation theory for the present case. As will be shown below, at the one-loop level everything works fine and on physical grounds we expect this to happen at all orders.

Green's functions of the operators in Eq. (2.21) are, however, more singular at short distances than (gaugedependent) Green's functions of the fields $\Phi, W_{\mu}^{a}$, and $B_{\mu}$ themselves. Time ordering of these operators gives rise to ambiguities, and the corresponding Green's functions are only unique up to contact terms. In order to make the theory finite, these contact terms of dimension four need to be added to the Lagrangian which is then given by

$$
\mathcal{L}_{\mathrm{SM}}=\mathcal{L}_{\mathrm{SM}}^{0}+\widehat{\mathcal{L}}_{\text {source }}^{1}+\mathcal{L}_{\text {source }}^{2} .
$$

The first term in Eq. 2.25) is defined in Eq. (2.17). The second term is given by

$$
\widehat{\mathcal{L}}_{\text {source }}^{1}=-\frac{1}{2} \widehat{h} \Phi^{\dagger} \Phi-\frac{1}{2} \widehat{K}_{\mu \nu} B_{\mu \nu}+J_{\mu}^{a} \varphi^{a b} V_{\mu}^{b},
$$

where

$$
\begin{aligned}
\widehat{h} & =h+4 v_{j j} J_{\mu}^{+} J_{\mu}^{-}+c_{j j} J_{\mu}^{\mathcal{Z}} J_{\mu}^{\mathcal{Z}}+4 J_{\mu}^{a} J_{\mu}^{a}, \\
\widehat{K}_{\mu \nu} & =K_{\mu \nu}+c_{B j}\left(\partial_{\mu} J_{\nu}^{\mathcal{Z}}-\partial_{\nu} J_{\mu}^{\mathcal{Z}}\right)-2 i c_{B j j}\left(J_{\mu}^{+} J_{\nu}^{-}-J_{\mu}^{-} J_{\nu}^{+}\right) .
\end{aligned}
$$

The last term in Eq. (2.25) is defined by

$$
\begin{aligned}
\mathcal{L}_{\text {source }}^{2}= & -v_{d j j} J_{\nu}^{\mathcal{Z}}\left[i\left(d_{\mu} J_{\nu}^{+}-d_{\nu} J_{\mu}^{+}\right) J_{\mu}^{-}-i\left(d_{\mu} J_{\nu}^{-}-d_{\nu} J_{\mu}^{-}\right) J_{\mu}^{+}\right]+v_{d j}\left(d_{\mu} J_{\nu}^{+}-d_{\nu} J_{\mu}^{+}\right)\left(d_{\mu} J_{\nu}^{-}-d_{\nu} J_{\mu}^{-}\right) \\
& -\frac{i}{2} c_{d j j}\left(\partial_{\mu} J_{\nu}^{\mathcal{Z}}-\partial_{\nu} J_{\mu}^{\mathcal{Z}}\right)\left(J_{\mu}^{+} J_{\nu}^{-}-J_{\mu}^{-} J_{\nu}^{+}\right)+\frac{1}{4} c_{d j}\left(\partial_{\mu} J_{\nu}^{\mathcal{Z}}-\partial_{\nu} J_{\mu}^{\mathcal{Z}}\right)\left(\partial_{\mu} J_{\nu}^{\mathcal{Z}}-\partial_{\nu} J_{\mu}^{\mathcal{Z}}\right) \\
& +16 v_{J J 2}\left(J_{\mu}^{+} J_{\mu}^{-}\right)^{2}+4 v_{J J J J}\left(J_{\mu}^{+} J_{\nu}^{-}+J_{\mu}^{-} J_{\nu}^{+}\right)^{2}+c_{J J 2}\left(J_{\mu}^{\mathcal{Z}} J_{\mu}^{\mathcal{Z}}\right)^{2} \\
& +4 v_{J 2 Z Z} J_{\mu}^{+} J_{\mu}^{-} J_{\nu}^{\mathcal{Z}} J_{\nu}^{\mathcal{Z}}+2 v_{J J Z Z}\left(J_{\mu}^{+} J_{\nu}^{-}+J_{\mu}^{-} J_{\nu}^{+}\right) J_{\mu}^{\mathcal{Z}} J_{\nu}^{\mathcal{Z}} \\
& +c_{h h} h^{2}+c_{m h} m^{2} h+4 c_{h J J} h J_{\mu}^{+} J_{\mu}^{-}+4 c_{m J J} m^{2} J_{\mu}^{+} J_{\mu}^{-}+c_{h Z Z} h J_{\mu}^{\mathcal{Z}} J_{\mu}^{\mathcal{Z}}+c_{m Z Z} m^{2} J_{\mu}^{\mathcal{Z}} J_{\mu}^{\mathcal{Z}},
\end{aligned}
$$

where we introduced the quantities

$$
\begin{aligned}
J_{\mu}^{ \pm} & =\frac{1}{2}\left(J_{\mu}^{1} \mp i J_{\mu}^{2}\right), \quad J_{\mu}^{\mathcal{Z}} \equiv J_{\mu}^{3}, \\
d_{\mu} J_{\nu}^{ \pm} & =\left(\partial_{\mu} \mp i B_{\mu}^{T}\right) J_{\nu}^{ \pm}, \quad B_{\mu}^{T}=\mathrm{PT}_{\mu \nu} B_{\nu} \\
\mathrm{PT}_{\mu \nu} & =\delta_{\mu \nu}-\mathrm{PL}_{\mu \nu}, \quad \mathrm{PL}_{\mu \nu}=\frac{\partial_{\mu} \partial_{\nu}}{\square} .
\end{aligned}
$$

The contact terms in $\mathcal{L}_{\text {source }}^{2}$ will not contribute to any physical $S$-matrix elements.

For later use we define the following $S U(2)_{L} \times U(1)_{Y}$ gauge-invariant fields: 


$$
\begin{aligned}
\underline{V}_{\mu}^{a} & =\varphi^{a b} V_{\mu}^{b}, \\
\mathcal{W}_{\mu}^{ \pm} & =\varphi^{\mp} \mathcal{W}_{\mu}^{ \pm}, \\
\mathcal{A}_{\mu}^{T} & =\mathrm{PT}_{\mu \nu} \mathcal{A}_{\nu},
\end{aligned}
$$

where

$$
\varphi^{\mp}(x)=\exp \left( \pm i \int d^{d} y \mathcal{G}_{0}(x-y) \partial_{\mu} B_{\mu}(y)\right) .
$$

The projection on the transverse mode in Eq. (2.35) leads to a fully $S U(2)_{L} \times U(1)_{Y}$ gauge-invariant field, since the $S U(2)_{L}$ invariant field $\mathcal{A}_{\mu}$ from Eq. (2.15) transforms under $U(1)_{Y}$ as follows:

$$
\mathcal{A}_{\mu} \rightarrow \mathcal{A}_{\mu}-\partial_{\mu} \omega
$$

i.e. like an Abelian gauge field.

Furthermore we introduce the quantities

$$
\begin{aligned}
\mathcal{Y}_{\mu}^{ \pm} & =\mathcal{W}_{\mu}^{ \pm}+4 j_{\mu}^{ \pm}, \mathcal{Y}_{\mu}^{\mathcal{Z}}=\mathcal{Z}_{\mu}+4 J_{\mu}^{\mathcal{Z}}, \\
j_{\mu}^{ \pm} & =\varphi^{ \pm} J_{\mu}^{ \pm} .
\end{aligned}
$$

The generating functional $W_{\mathrm{SM}}\left[h, K_{\mu \nu}, J_{\mu}^{a}\right]$ for the gauge-invariant Green's functions is defined by the path integral

$$
e^{-W_{\mathrm{SM}}\left[h, K_{\mu \nu}, J_{\mu}^{a}\right]}=\int \mathrm{d} \mu\left[\Phi, W_{\mu}^{a}, B_{\mu}\right] e^{-\int \mathrm{d}^{d} x \mathcal{L}_{\mathrm{SM}}} .
$$

Note that we still integrate over the original fields $\Phi, W_{\mu}^{a}$, and $B_{\mu}$ in Eq. (2.40). Furthermore, we have absorbed an appropriate normalization factor into the measure $\mathrm{d} \mu\left[\Phi, W_{\mu}^{a}, B_{\mu}\right]$. Derivatives of this functional with respect to the field $h$ generate Green's functions of the scalar density $\Phi^{\dagger} \Phi$, derivatives with respect to the source $K_{\mu \nu}$ generate Green's functions of the field strength $B_{\mu \nu}$, while derivatives with respect to $J_{\mu}^{a}$ generate Green's functions for the currents $\underline{V}_{\mu}^{a}$.

In the spontaneously broken phase, these Green's functions have one-particle poles from the Higgs boson as well as the gauge bosons. Thus, one can extract $S$-matrix elements for the physical degrees of freedom from the generating functional in Eq. (2.40). Due to the equivalence theorem [28] these $S$-matrix elements will be identical to the ones obtained from those Green's functions which are used in the usually employed formalism. The presence of the contact terms in $\mathcal{L}_{\text {source }}^{2}$ in Eq. (2.29) reflects the fact that the off-shell continuation of the $S$-matrix is not unambiguously defined. Note that this is a general feature of any field theory and not particular to those involving a gauged symmetry. The continuation we choose here has the virtue of being gauge-invariant.

There is another aspect worth noting. In Refs. 225,26] it was pointed out that the complete screening of the $S U(2)_{L}$ charge of the composite fields $V_{\mu}^{a}$ and $\Phi^{\dagger} \Phi$ can be interpreted as the manifestation of confinement in the electroweak theory, similarly to the mechanism in QCD. As discussed in Ref. 26] the physically observed particles then correspond to "mesonic" and "baryonic" bound states of the usual fields that appear in the Lagrangian. To illustrate this point more clearly, it is useful to include the fermions for a moment. As shown in Ref. [17], for up and down type quarks and leptons one may consider the following composite fields:

$$
\begin{array}{rlrl}
\Phi^{\dagger} q_{L}^{k} & =\frac{m}{\sqrt{\lambda}} R d_{L}^{k}, & \widetilde{\Phi}^{\dagger} q_{L}^{k}=\frac{m}{\sqrt{\lambda}} R u_{L}^{k}, \\
\Phi^{\dagger} l_{L}^{k}=\frac{m}{\sqrt{\lambda}} R e_{L}^{k}, & \widetilde{\Phi}^{\dagger} l_{L}^{k}=\frac{m}{\sqrt{\lambda}} R \nu_{L}^{k},
\end{array}
$$

which appear in the Yukawa interactions. The interpolating fermion fields

$$
\begin{array}{rlrl}
d_{L}^{k} & =U^{\dagger} q_{L}^{k}, & & u_{L}^{k}=\widetilde{U}^{\dagger} q_{L}^{k}, \\
e_{L}^{k} & =U^{\dagger} l_{L}^{k}, & \nu_{L}^{k}=\widetilde{U}^{\dagger} l_{L}^{k},
\end{array}
$$

are $S U(2)_{L}$-invariant and have the same $U(1)_{Y}$ quantum numbers as their right-handed counterparts. The fields $q_{L}^{k}$ and $l_{L}^{k}$ in Eqs. (2.41) and (2.42) are the usual fermion doublets for the quarks and leptons with family index $k$.

Denoting schematically all doublets (Higgs, quarks, leptons) by $q$, one can interpret the fields built out of $q^{\dagger} q^{\prime}$ as "mesons" and the fields $\tilde{q}^{\dagger} q^{\prime} \equiv-q_{i} \epsilon^{i j} q_{j}^{\prime}$ as "baryons," cf. Eq. (2.10). The fields $q^{\dagger} D_{\mu} q^{\prime}$ and $\tilde{q}^{\dagger} D_{\mu} q^{\prime}$ can then be 
viewed at as $P$-wave states of these "mesons" and "baryons." Thus we have the following "mesons" $R d_{L}^{k}, R e_{L}^{k}, R^{2} \mathcal{Z}_{\mu}$ and $\Phi^{\dagger} \Phi$, which correspond for each family $k$ to the physical $d$-quark , the electron, the $Z$-boson and to the Higgs boson, respectively. Furthermore, there are the following "baryons" $R u_{L}^{k}, R \nu_{L}^{k}$ and $R^{2} \mathcal{W}_{\mu}^{ \pm}$, which correspond to the physical $u$-quark, the neutrino and the $W$-bosons. The fundamental fields $l_{L}^{k}, q_{L}^{k}$ and $\Phi$ which carry $S U(2)_{L}$-charges are confined at low energies, i.e. around the electroweak scale, in these "mesons" and "baryons" due to the strong non-Abelian forces of the $S U(2)_{L}$ gauge fields. Therefore the $S U(2)_{L}$ charge cannot be observed in physical states, similarly to color in QCD. Note that the notions "meson" and "baryon" are convention dependent. In particular, we use different conventions than those employed in Ref. [26].

Our approach, extending the gauge-invariant treatment to the full group $S U(2)_{L} \times U(1)_{Y}$, can thus be viewed at as a well-defined framework for carrying out calculations which involve only those external fields which correspond to the physically observed particles.

As was pointed out in Refs. 10,17] it is possible to evaluate the path integral in Eq. (2.40) without the need to fix a gauge as will be shown in the following.

\section{TREE LEVEL}

At tree level, the generating functional for the bosonic sector of the standard model is given by

$$
W_{\mathrm{SM}}\left[h, K_{\mu \nu}, J_{\mu}^{a}\right]=\int \mathrm{d}^{d} x \mathcal{L}_{\mathrm{SM}}\left(R^{c l}, \underline{\mathcal{W}}_{\mu}^{c l, \pm}, \mathcal{Z}_{\mu}^{c l}, \mathcal{A}_{\mu}^{c l, T}\right)
$$

where $R^{c l}, \underline{\mathcal{W}}_{\mu}^{c l, \pm}, \mathcal{Z}_{\mu}^{c l}$, and $\mathcal{A}_{\mu}^{c l, T}$ are determined by the equations of motion

$$
\begin{aligned}
-\square R & =-\left[m^{2}\left(R^{2}-1\right)+\underline{\mathcal{Y}}_{\mu}^{+} \underline{\mathcal{Y}}_{\mu}^{-}+\frac{1}{4} \mathcal{Y}_{\mu}^{\mathcal{Z}} \mathcal{Y}_{\mu}^{\mathcal{Z}}-\widehat{h}\right] R, \\
-\underline{d}_{\mu} \underline{\mathcal{W}}_{\mu \nu}^{ \pm} & =-M_{W}^{2} R^{2}\left(\underline{\mathcal{W}}_{\nu}^{ \pm}+4 J_{\nu}^{ \pm}\right) \pm i\left(c^{2} \mathcal{Z}_{\mu \nu}+\mathcal{A}_{\mu \nu}\right) \underline{\mathcal{W}}_{\mu}^{ \pm} \pm 2\left(\underline{\mathcal{W}}_{\mu}^{+} \underline{\mathcal{W}}_{\nu}^{-}-\underline{\mathcal{W}}_{\mu}^{-} \underline{\mathcal{W}}_{\nu}^{+}\right) \underline{\mathcal{W}}_{\mu}^{ \pm}, \\
-\partial_{\mu} \mathcal{Z}_{\mu \nu} & =\mathrm{PT}_{\nu \mu}\left(-M_{Z}^{2} R^{2} \mathcal{Y}_{\mu}^{\mathcal{Z}}+T_{\mu}\right)+\frac{e^{2}}{c^{2}} \partial_{\mu} \widehat{K}_{\mu \nu}+\frac{e^{2}}{c^{2}} \operatorname{PT}_{\nu \mu} S_{\mu}, \\
-\partial_{\mu} \mathcal{A}_{\mu \nu} & =s^{2} \mathrm{PT}_{\nu \mu} T_{\mu}-e^{2} \partial_{\mu} \widehat{K}_{\mu \nu}-e^{2} \mathrm{PT}_{\nu \mu} S_{\mu} .
\end{aligned}
$$

Furthermore, the equations for the Goldstone boson field $U$ correspond to

$$
\begin{aligned}
& \underline{d}_{\mu} \underline{\mathcal{Y}}_{\mu}^{ \pm}=-2 \frac{\partial_{\mu} R}{R} \mathcal{Y}_{\mu}^{ \pm} \mp i \mathcal{Y}_{\mu}^{\mathcal{Z}} \underline{\mathcal{W}}_{\mu}^{ \pm}, \\
& \partial_{\mu} \mathcal{Y}_{\mu}^{\mathcal{Z}}=-2 \frac{\partial_{\mu} R}{R} \mathcal{Y}_{\mu}^{\mathcal{Z}}-8 i\left(J_{\mu}^{+} \underline{\mathcal{W}}_{\mu}^{-}-J_{\mu}^{-} \underline{\mathcal{W}}_{\mu}^{+}\right) .
\end{aligned}
$$

In order to simplify the notation we have omitted the prescription "cl" in the equations above. In Eqs. (3.2)-(3.7) we have introduced the quantities

$$
\begin{aligned}
\underline{\mathcal{Y}}_{\mu}^{ \pm}= & \varphi^{\mp} \mathcal{Y}_{\mu}^{ \pm}=\underline{\mathcal{W}}_{\mu}^{ \pm}+4 J_{\mu}^{ \pm} \\
\underline{d}_{\mu} \underline{\mathcal{W}}_{\nu}^{ \pm}= & \left(\partial_{\mu} \mp i\left[\mathcal{Z}_{\mu}+s^{2} \mathcal{Z}_{\mu}^{T}-\mathcal{A}_{\mu}^{T}\right]\right) \underline{\mathcal{W}}_{\nu}^{ \pm} \\
\underline{\mathcal{W}}_{\mu \nu}^{ \pm}= & \underline{d}_{\mu} \underline{\mathcal{W}}_{\nu}^{ \pm}-\underline{d}_{\nu} \underline{\mathcal{W}}_{\mu}^{ \pm} \\
\mathcal{Z}_{\mu \nu}= & \partial_{\mu} \mathcal{Z}_{\nu}-\partial_{\nu} \mathcal{Z}_{\mu} \\
\mathcal{A}_{\mu \nu}= & \partial_{\mu} \mathcal{A}_{\nu}-\partial_{\nu} \mathcal{A}_{\mu} \\
T_{\mu}= & 2 \mathcal{Z}_{\rho}\left(\underline{\mathcal{W}}_{\rho}^{+} \underline{\mathcal{W}}_{\mu}^{-}+\underline{\mathcal{W}}_{\mu}^{+} \underline{\mathcal{W}}_{\rho}^{-}\right)-4 \mathcal{Z}_{\mu} \underline{\mathcal{W}}_{\rho}^{+} \underline{\mathcal{W}}_{\rho}^{-}+2 i\left(\underline{\mathcal{W}}_{\rho \mu}^{+} \underline{\mathcal{W}}_{\rho}^{-}-\underline{\mathcal{W}}_{\rho \mu}^{-} \underline{\mathcal{W}}_{\rho}^{+}\right) \\
& -2 i\left(\underline{d}_{\rho} \underline{\mathcal{W}}_{\rho}^{+} \underline{\mathcal{W}}_{\mu}^{-}-\underline{d}_{\rho} \underline{\mathcal{W}}_{\rho}^{-} \underline{\mathcal{W}}_{\mu}^{+}-\underline{d}_{\rho} \underline{\mathcal{W}}_{\mu}^{+} \underline{\mathcal{W}}_{\rho}^{-}+\underline{d}_{\rho} \underline{\mathcal{W}}_{\mu}^{-} \underline{\mathcal{W}}_{\rho}^{+}\right) \\
S_{\mu}= & -v_{d j j} J_{\rho}^{\mathcal{Z}}\left(J_{\rho}^{+} J_{\mu}^{-}+J_{\rho}^{-} J_{\mu}^{+}\right)+2 v_{d j j} J_{\mu}^{\mathcal{Z}} J_{\rho}^{+} J_{\rho}^{-} \\
& -2 v_{d j}\left[i\left(d_{\rho} J_{\mu}^{+}-d_{\mu} J_{\rho}^{+}\right) J_{\rho}^{-}-i\left(d_{\rho} J_{\mu}^{-}-d_{\mu} J_{\rho}^{-}\right) J_{\rho}^{+}\right]
\end{aligned}
$$

\footnotetext{
${ }^{2}$ Of course, if we switch on the QCD interactions, the quarks will be confined in hadrons.
} 
The covariant derivatives in $d_{\mu} J_{\mu}^{ \pm}, \underline{d}_{\mu} \mathcal{Y}_{\nu}^{ \pm}$, and $\underline{d}_{\mu} \underline{\mathcal{W}}_{\mu \nu}^{ \pm}$are defined in the same way as in Eq. (3.9).

Several things about Eqs. (3.2)-(3.7) are worth being noticed. First of all, only the physical degrees of freedom enter these equations. The radial variable $R$ which is related to the massive Higgs boson is determined by Eq. (3.2). Solutions for the massive gauge boson fields $\underline{\mathcal{W}}_{\mu}^{ \pm}$, cf. Eq. (2.34), and $\mathcal{Z}_{\mu}$ follow from Eqs. (3.3) and (3.4). Finally, Eq. (3.5) determines the transverse component of the massless photon field $\mathcal{A}_{\mu}^{T}$. Note that the equations of motion do not determine the longitudinal component of the photon field and the phase of the gauge boson fields $\mathcal{W}_{\mu}^{ \pm}$which correspond to the $U(1)_{Y}$ gauge degree of freedom. Even more they do not determine the classical Goldstone boson field $U$ either, since it corresponds to the $S U(2)_{L}$ gauge degrees of freedom. Thus, gauge invariance implies that these equations have a whole class of solutions in terms of the original fields $\Phi, W_{\mu}^{a}, B_{\mu}$. Every two representatives are related to each other by a gauge transformation. Nevertheless, the physical degrees of freedom are uniquely determined by these equations of motion. Moreover, since the action is gauge-invariant, the generating functional in Eq. (3.1) is uniquely determined as well.

The most important point is the fact that the classical Goldstone boson field $U$ represents the $S U(2)_{L}$ gauge degrees of freedom. Thus, no Goldstone bosons are propagating at the classical level of the theory. All gauge-invariant sources emit physical modes only. Moreover, Eqs. (3.6) and (3.7), which follow from the requirement that the variation of the Lagrangian with respect to the Goldstone boson field $U$ vanishes, are not equations of motion, but constraints expressing the fact that the gauge fields $\underline{\mathcal{W}}_{\mu}^{ \pm}, \mathcal{Z}_{\mu}$, and $\mathcal{A}_{\mu}$ couple to conserved currents. They can also be obtained by taking the derivative of the equations of motion for the gauge fields. Note that we have already used the constraints to bring these equations of motion into the form given in Eqs. (3.2)-(3.5).

In order to solve the classical equations of motion (3.2)-(3.5) we introduce a parameter $\chi$ which counts powers of the external sources:

$$
h, K_{\mu \nu}, J_{\mu}^{a}=\mathcal{O}(\chi) .
$$

From this we get the counting rules

$$
R-1, \underline{\mathcal{W}}_{\mu}^{ \pm}, \mathcal{Z}_{\mu}, \mathcal{A}_{\mu}=\mathcal{O}(\chi) .
$$

We will see below that this counting scheme is self-consistent.

The solution of the equation of motion for the Higgs field $R$, Eq. (3.2), reads, up to and including quadratic terms in powers of the external sources,

$$
\begin{aligned}
(R-1)(x)= & \int d^{d} y \mathcal{G}_{H}(x-y)\left(\widehat{h}-\mathcal{Y}_{\mu}^{+} \mathcal{Y}_{\mu}^{-}-\frac{1}{4} \mathcal{Y}_{\mu}^{\mathcal{Z}} \mathcal{Y}_{\mu}^{\mathcal{Z}}\right)(y)+\int d^{d} y d^{d} z \mathcal{G}_{H}(x-y)\left(h(y) \mathcal{G}_{H}(y-z) h(z)\right) \\
& -3 m^{2} \int d^{d} y \mathcal{G}_{H}(x-y)\left(\int d^{d} z \mathcal{G}_{H}(y-z) h(z)\right)^{2} .
\end{aligned}
$$

In order to calculate the two-point functions of the physical fields in Sec. VI we will not need the terms of third and higher order in powers of the external sources in Eq. (3.17).

The solutions for the equations of motion for the gauge fields, Eqs. (3.3)-(3.5), are given by

$$
\begin{aligned}
\underline{\mathcal{W}}_{\mu}^{ \pm, T}(x) & =\int d^{d} y \mathcal{G}_{W}(x-y)\left(-4 M_{W}^{2} J_{\mu}^{ \pm, T}\right)(y), \\
\underline{\mathcal{W}}_{\mu}^{ \pm, L}(x) & =-4 J_{\mu}^{ \pm, L}(x) \\
\mathcal{Z}_{\mu}^{T}(x) & =\int d^{d} y \mathcal{G}_{Z}(x-y)\left(-4 M_{Z}^{2} J_{\mu}^{\mathcal{Z}, T}+\frac{e^{2}}{c^{2}} \partial_{\rho} \widehat{K}_{\rho \mu}\right)(y), \\
\mathcal{Z}_{\mu}^{L}(x) & =-4 J_{\mu}^{\mathcal{Z}, L}(x), \\
\mathcal{A}_{\mu}^{T}(x) & =\int d^{d} y \mathcal{G}_{A}(x-y)\left(-e^{2} \partial_{\rho} \widehat{K}_{\rho \mu}\right)(y) .
\end{aligned}
$$

We will only need the leading terms of the solution in powers of the external sources later on. In Eqs. (3.17)-(3.22) we have introduced the quantities

$$
\begin{array}{ll}
J_{\mu}^{ \pm, T}=\mathrm{PT}_{\mu \nu} J_{\nu}^{ \pm}, & J_{\mu}^{\mathcal{Z}, T}=\mathrm{PT}_{\mu \nu} J_{\nu}^{\mathcal{Z}}, \\
J_{\mu}^{ \pm, L}=\mathrm{PL}_{\mu \nu} J_{\nu}^{ \pm}, & J_{\mu}^{\mathcal{Z}, L}=\mathrm{PL}_{\mu \nu} J_{\nu}^{\mathcal{Z}},
\end{array}
$$

and 


$$
\begin{aligned}
\mathcal{G}_{H}(x-y) & =\left\langle x\left|\frac{1}{-\square+2 m^{2}}\right| y\right\rangle, \\
\mathcal{G}_{W}(x-y) & =\left\langle x\left|\frac{1}{-\square+M_{W}^{2}}\right| y\right\rangle, \\
\mathcal{G}_{Z}(x-y) & =\left\langle x\left|\frac{1}{-\square+M_{Z}^{2}}\right| y\right\rangle, \\
\mathcal{G}_{A}(x-y) & =\left\langle x\left|\frac{1}{-\square}\right| y\right\rangle .
\end{aligned}
$$

The $U(1)_{Y}$ gauge degree of freedom of the longitudinal component of $B_{\mu}^{L}$ can be parametrized as follows:

$$
B_{\mu}^{L}=\partial_{\mu} \omega,
$$

with an arbitrary function $\omega$. The solutions for the longitudinal component of the photon $\mathcal{A}_{\mu}^{L}$ and the phase of the $W$-boson field $\mathcal{W}_{\mu}^{ \pm}$are given by

$$
\begin{aligned}
\mathcal{A}_{\mu}^{L} & =\partial_{\mu} \omega+s^{2} \mathcal{Z}_{\mu}^{L}, \\
\mathcal{W}_{\mu}^{ \pm} & =e^{ \pm i \omega} \underline{\mathcal{W}}_{\mu}^{ \pm} .
\end{aligned}
$$

The gauge dependence of these fields manifests itself through the presence of the undetermined function $\omega$. The $S U(2)_{L}$ gauge invariance corresponds to the freedom to choose an arbitrary field $U$. For instance, the choice $U=\left(\begin{array}{l}0 \\ 1\end{array}\right)$ leads to the unitary gauge.

\section{ONE-LOOP LEVEL}

The one-loop contribution to the generating functional can be evaluated with the saddle-point method. Before we proceed with the explicit calculation of the generating functional some general remarks are in order. If we write the fluctuations $y$ around the classical fields $\mathcal{F}^{c l}$ as $\mathcal{F}=\mathcal{F}^{c l}+y$, we obtain the following representation for the one-loop approximation to the generating functional:

$$
e^{-W_{\mathrm{SM}}\left[h, K_{\mu \nu}, J_{\mu}^{a}\right]}=e^{-\int \mathrm{d}^{d} x \mathcal{L}_{\mathrm{SM}}^{c l}} \int \mathrm{d} \mu[y] e^{-(1 / 2) \int \mathrm{d}^{d} x y^{T} \widetilde{D} y} .
$$

Gauge invariance implies that the operator $\widetilde{D}$ has zero eigenvalues corresponding to fluctuations $y$ which are equivalent to infinitesimal gauge transformations. Indeed, if $\mathcal{F}^{c l, i}$ is a solution of the equation of motion, i.e., a stationary point of the classical action,

$$
\left.\frac{\delta S_{\mathrm{SM}}}{\delta \mathcal{F}^{i}}\right|_{\mathcal{F}=\mathcal{F}^{c l}}=0
$$

then any gauge transformation yields another equivalent solution. The index $i$ in $\mathcal{F}^{c l, i}$ labels the different fields. Thus, differentiating equation (4.2) with respect to the gauge parameters $\omega^{A}$ one obtains

$$
\left.\frac{\delta^{2} S_{\mathrm{SM}}}{\delta \mathcal{F}^{i} \delta \mathcal{F}^{j}} \frac{\delta \mathcal{F}^{j}}{\delta \omega^{A}}\right|_{\mathcal{F}_{=\mathcal{F}} c l}=0
$$

The quadratic form which appears in Eq. (4.3) is identical to the differential operator $\widetilde{D}$. If we denote the zero eigenvector by $\zeta$ and parametrize it in terms of scalar fields $\alpha$ by way of $\zeta=P \alpha$, with some differential operator $P$, Eq. (4.3) translates to the identity $P^{T} \widetilde{D}=\widetilde{D} P=0$. Let $\alpha_{m}$ be the eigenvectors of the operator $P^{T} P$, i.e.

$$
P^{T} P \alpha_{m}=l_{m} \alpha_{m} .
$$

Then, the expansion of the fluctuation $y$ in terms of eigenvectors of the operator $\widetilde{D}$ is given by

$$
y=\sum_{n} a_{n} \xi_{n}+\sum_{m} b_{m} \zeta_{m},
$$


where $\zeta_{m}=P \alpha_{m}$ and $\xi_{n}$ have zero and non-zero eigenvalues, respectively.

In order to evaluate the path integral in Eq. (4.1), we use Polyakov's method 29] and equip the space of fields with a metric

$$
\|y\|^{2}=\int \mathrm{d}^{d} x y^{T} y=\sum_{n} a_{n}^{2}+\sum_{m} b_{m}^{2} l_{m}
$$

With our choice for the scalar fields $\alpha_{m}$, the metric on the kernel of the differential operator $\widetilde{D}$ is diagonal:

$$
g_{\bar{m} m}=\int \mathrm{d}^{d} x \alpha_{\bar{m}} P^{T} P \alpha_{m}=\delta_{\bar{m} m} l_{m},
$$

and the volume element associated with this metric is then given by

$$
\mathrm{d} \mu\left[\Phi, W_{\mu}^{a}, B_{\mu}\right]=\mathcal{N} \prod_{n} \mathrm{~d} a_{n} \prod_{m} \mathrm{~d} b_{m} \sqrt{\operatorname{det} P^{T} P} .
$$

The integration over the zero modes yields the volume factor of the gauge group, which can be absorbed by the normalization of the integral. The remaining integral over the non-zero modes is damped by the usual Gaussian factor. Up to an irrelevant infinite constant one obtains the following result for the one-loop generating functional from Eq. 4.1):

$$
W_{\mathrm{SM}}\left[h, K_{\mu \nu}, J_{\mu}^{a}\right]=\int \mathrm{d}^{d} x \mathcal{L}_{\mathrm{SM}}+\frac{1}{2} \ln \operatorname{det}^{\prime} \widetilde{D}-\frac{1}{2} \ln \operatorname{det} P^{T} P .
$$

The first term on the right-hand side represents the classical action which describes the tree-level contributions to the generating functional. In the second term, the determinant $\operatorname{det}^{\prime} \widetilde{D}$ is defined as the product of all non-zero eigenvalues of the operator $\widetilde{D}$. The last term originates from the path integral measure. The sum of the last two terms in Eq. (4.9) corresponds to the one-loop contributions to the generating functional.

We now discuss in more detail the evaluation of the one-loop contributions to the generating functional in Eq. (4.1) for the standard model. The choice of an appropriate parametrization of the physical modes and their quantum fluctuations is very important in order to obtain an expression for the differential operator which is still tractable. We introduce the following fluctuations $f, \eta^{a}, w_{\mu}^{a}$, and $b_{\mu}$ around the Higgs field $R$, the Goldstone boson field $U$, the three $S U(2)_{L}$ gauge fields $W_{\mu}^{a}$ and the $U(1)_{Y}$ gauge field $B_{\mu}$, respectively:

$$
\begin{aligned}
R & \rightarrow R+\frac{\sqrt{\lambda}}{m} f \\
U & \rightarrow e^{i \kappa / 2} V U \\
W_{\mu} & \rightarrow W_{\mu}+\frac{1}{2} g w_{\mu}^{a} V t^{a} V^{\dagger}, \quad W_{\mu} \equiv W_{\mu}^{a} \frac{\tau^{a}}{2}, \\
B_{\mu} & \rightarrow B_{\mu}+g^{\prime} b_{\mu}
\end{aligned}
$$

where

$$
\begin{aligned}
\kappa(x) & =-g^{\prime} \int \mathrm{d}^{d} y \mathcal{G}_{0}(x-y) \partial_{\mu} b_{\mu}(y), \\
V(x) & =\exp \left(i \frac{\sqrt{\lambda}}{m R(x)} \eta^{a}(x) t^{a}(x)\right), \quad V \in S U(2) .
\end{aligned}
$$

The matrices $t^{i}$ in Eq. (4.15) are defined through the relations

$$
\begin{aligned}
& t^{1}=U \widetilde{U}^{\dagger}+\widetilde{U} U^{\dagger}, \\
& t^{2}=i\left(U \widetilde{U}^{\dagger}-\widetilde{U} U^{\dagger}\right), \\
& t^{3}=\widetilde{U} \widetilde{U}^{\dagger}-U U^{\dagger},
\end{aligned}
$$

and satisfy the Pauli algebra

$$
\left[t^{i}, t^{j}\right]=2 i \varepsilon^{i j k} t^{k}, \quad\left\{t^{i}, t^{j}\right\}=2 \delta^{i j} \mathbf{1}
$$


Using the above transformation properties we get

$$
\begin{aligned}
\mathcal{W}_{\mu}^{a} & \rightarrow\left(e^{T \kappa}\right)^{a b}\left(\mathcal{W}_{\mu}^{b}+g w_{\mu}^{b}+g^{\prime} \mathrm{PL}_{\mu \nu} b_{\nu} \delta^{b 3}+i \operatorname{tr}\left(t^{b} V^{\dagger} D_{\mu} V\right)\right) \\
& =\left(e^{T \kappa}\right)^{a b}\left(\mathcal{W}_{\mu}^{b}+g w_{\mu}^{b}+g^{\prime} \mathrm{PL}_{\mu \nu} b_{\nu} \delta^{b 3}-\frac{2 \sqrt{\lambda}}{m}\left(\mathcal{D}_{\mu} \frac{\eta}{R}\right)^{b}-\frac{2 \lambda}{m^{2}} \varepsilon^{b c d} \frac{\eta^{c}}{R}\left(\mathcal{D}_{\mu} \frac{\eta}{R}\right)^{d}\right)
\end{aligned}
$$

where

$$
\mathcal{D}_{\mu}^{a b} \eta^{b}=\partial_{\mu} \eta^{a}-\varepsilon^{a b c} \mathcal{W}_{\mu}^{c} \eta^{b}
$$

The basic idea for this choice of parametrization of the quantum fluctuations is the following. If we shift the fields only linearly, the correspondence between zero modes of the differential operator and fluctuations corresponding to gauge transformations is only true at leading order. However, if we use the parametrization given above this correspondence is true at higher orders in the fluctuations as well.

We collect all the fluctuations in a vector

$$
y=\left(\begin{array}{c}
f \\
\eta^{a} \\
q_{\mu}^{A}
\end{array}\right)
$$

where $q_{\mu}^{A} \doteq\left(\begin{array}{c}w_{\mu}^{a} \\ b_{\mu}\end{array}\right)$ describes the fluctuations of the gauge fields. Here and in the following, lowercase Latin indices $(a, b)$ run from 1 to 3 , whereas uppercase Latin indices $(A, B)$ run from 1 to 4 . The differential operator $\widetilde{D}$ which is acting on the space of fluctuations from Eq. (4.20) can be represented by a $3 \times 3$-matrix. Before we write down this matrix it is useful to make some additional transformations of the differential operator.

As noted above, the differential operator $\widetilde{D}$ has zero modes due to gauge invariance. In the basis $f, \eta^{a}, q_{\mu}^{A}$ they can be written in the form

$$
\left(\begin{array}{c}
0 \\
M_{W} R \delta^{a B} \\
\widetilde{\mathcal{D}}_{\mu}^{A B}
\end{array}\right) \alpha^{B} \equiv P \alpha
$$

where $\alpha^{B}$ are four arbitrary scalar functions. The covariant derivative $\widetilde{\mathcal{D}}_{\mu}^{A B}$ is defined through

$$
\begin{aligned}
& \widetilde{\mathcal{D}}_{\mu}^{A B}=\delta^{A B} \partial_{\mu}-f^{A B c} \mathcal{W}_{\mu}^{c}, \\
& f^{A B c}=\left\{\begin{array}{cl}
\varepsilon^{a b c}, & A=a, B=b, \\
0, & A=4 \text { and } / \text { or } B=4 .
\end{array}\right.
\end{aligned}
$$

The generating functional is then given by the expression in Eq. (4.9). Using the fact that zero and non-zero eigenvectors are orthogonal to each other leads to the identity

$$
\ln \operatorname{det}^{\prime} \widetilde{D}=\ln \operatorname{det}\left(\widetilde{D}+P P^{T}+\delta_{P}\right)-\ln \operatorname{det}\left(P^{T} P\right),
$$

up to an irrelevant infinite constant. Again, $\operatorname{det}^{\prime} \widetilde{D}$ denotes the product of all non-zero eigenvalues. The operator $\delta_{P}$ will be defined below.

In order to remove the dependence of the differential operators $\widetilde{D}+P P^{T}+\delta_{P}$ and $P^{T} P$ on the phase factor $\varphi$ we define

$$
\begin{aligned}
\underline{\widetilde{D}}+\underline{P P^{T}}+\delta_{P} & \doteq O_{1}\left(\widetilde{D}+P P^{T}+\delta_{P}\right) O_{1}^{T}, \\
\underline{P^{T} P} & \doteq O_{2}\left(P^{T} P\right) O_{2}^{T},
\end{aligned}
$$

where

$$
O_{1}=\left(\begin{array}{ccc}
1 & 0 & 0 \\
0 & \varphi^{a b} & 0 \\
0 & 0 & O_{2}^{A B}
\end{array}\right), \quad O_{2}=\left(\begin{array}{cc}
\varphi^{a b} & 0 \\
0 & 1
\end{array}\right)
$$


The transformation matrices $O_{1}$ and $O_{2}$ have unit determinant since $\operatorname{det} \varphi=1$, because the matrix $T$ which appears in the phase factor is traceless, cf. Eq. (2.23).

Therefore, the generating functional at the one-loop level can be written in the following form:

$$
W_{\mathrm{SM}}\left[h, K_{\mu \nu}, J_{\mu}^{a}\right]=\int \mathrm{d}^{d} x \mathcal{L}_{\mathrm{SM}}+\frac{1}{2} \ln \operatorname{det}\left(\underline{\widetilde{D}}+\underline{P P^{T}}+\delta_{P}\right)-\ln \operatorname{det} \underline{P^{T} P},
$$

where the solutions of the equations of motion (3.2)-(3.5) have to be inserted. It represents the full one-loop contributions of the bosonic sector of the standard model.

The explicit expressions for the components of the differential operator $\underline{\widetilde{D}}+\underline{P P^{T}}+\delta_{P}$, which we parametrize by

$$
\underline{\widetilde{D}}+\underline{P P^{T}}+\delta_{P} \doteq\left(\begin{array}{ccc}
d & \delta & \delta_{\nu} \\
\delta^{T} & D & \Delta_{\nu} \\
\delta_{\mu}^{T} & \Delta_{\mu}^{T} & D_{\mu \nu}
\end{array}\right),
$$

can be found in Eqs. (A1)-(A9) in Appendix A. The operators $\underline{P P^{T}}, \underline{P^{T} P}$, and $\delta_{P}$ are listed in Eqs. (A21) $-(\mathrm{A} 23)$.

We would like to stress an important point here. At the classical level only physical modes propagate. The classical Goldstone boson field $U^{c l}$ represents the $S U(2)_{L}$ gauge degrees of freedom. At the quantum level, however, the situation is different. Quantum fluctuations around the classical field $U^{c l}$ denoted by $\eta^{a}$, cf. Eqs. (4.11) and (4.15), imply virtual Goldstone boson modes propagating within loops. Note that these modes are absent in any gauge-dependent approach based on the unitary gauge. They are, however, necessary in order to ensure a decent high-energy behavior of the theory.

In order to diagonalize the differential operator $\underline{\widetilde{D}}+\underline{P P^{T}}+\delta_{P}$ we introduce some additional quantities:

$$
\begin{aligned}
\mathcal{D}_{\mu \nu} & =D_{\mu \nu}-\delta_{\mu}^{T} d^{-1} \delta_{\nu}-\vartheta_{\mu}^{T} \Theta^{-1} \vartheta_{\nu}, \\
\Theta & =D-\delta^{T} d^{-1} \delta, \\
\vartheta_{\nu} & =\Delta_{\nu}-\delta^{T} d^{-1} \delta_{\nu} .
\end{aligned}
$$

Using the identity

$$
\mathcal{T}^{T}\left(\underline{\widetilde{D}}+\underline{P P^{T}}+\delta_{P}\right) \mathcal{T}=\operatorname{diag}\left(\mathrm{d}, \Theta, \mathcal{D}_{\mu \nu}\right)
$$

where

$$
\mathcal{T}=\left(\begin{array}{ccc}
1 & -d^{-1} \delta & -d^{-1} \delta_{\nu}+d^{-1} \delta \Theta^{-1} \vartheta_{\nu} \\
0 & 1 & -\Theta^{-1} \vartheta_{\nu} \\
0 & 0 & \delta_{\mu \nu}
\end{array}\right),
$$

and the fact that the transformation matrix $\mathcal{T}$ has unit determinant, one obtains the following intermediate result for the generating functional:

$$
W_{\mathrm{SM}}\left[h, K_{\mu \nu}, J_{\mu}^{a}\right]=\int \mathrm{d}^{d} x \mathcal{L}_{\mathrm{SM}}+\frac{1}{2} \ln \operatorname{det} d+\frac{1}{2} \ln \operatorname{det} \Theta+\frac{1}{2} \ln \operatorname{det} \mathcal{D}-\ln \operatorname{det} \underline{P^{T} P} .
$$

In a second step we rotate from the fluctuations of the weak eigenstates of the gauge bosons to the corresponding mass eigenstates:

$$
\mathcal{D}_{\mu \nu} \rightarrow \widetilde{\mathcal{D}}_{\mu \nu}=O \mathcal{D}_{\mu \nu} O^{T}
$$

with the orthogonal matrix

$$
O=\left(\begin{array}{cccc}
1 & 0 & 0 & 0 \\
0 & 1 & 0 & 0 \\
0 & 0 & c & -s \\
0 & 0 & s & c
\end{array}\right)
$$

After this rotation we get

$$
\widetilde{\mathcal{D}}_{\mu \nu}=\left(\begin{array}{ccc}
\widetilde{D}_{\mu \nu}^{\mathcal{W}} & \widetilde{\xi}_{\mu \nu}^{\mathcal{Z}} & \widetilde{\xi}_{\mu \nu}^{\mathcal{A}} \\
\widetilde{\xi}_{\mu \nu}^{\mathcal{Z}, T} & \widetilde{D}_{\mu \nu}^{\mathcal{Z}} & \widetilde{\eta}_{\mu \nu}^{\mathcal{A}} \\
\widetilde{\xi}_{\mu \nu}^{\mathcal{A}, T} & \widetilde{\eta}_{\mu \nu}^{\mathcal{A}, T} & \widetilde{D}_{\mu \nu}^{\mathcal{A}}
\end{array}\right)
$$


where the components are defined by the following equations:

$$
\begin{aligned}
\widetilde{D}_{\mu \nu}^{M} & =D_{\mu \nu}^{M}-\delta_{\mu}^{M, T} d^{-1} \delta_{\nu}^{M}-\vartheta_{\mu}^{M, T} \Theta^{-1} \vartheta_{\nu}^{M}, M=\mathcal{W}, \mathcal{Z}, \mathcal{A}, \\
\widetilde{\xi}_{\mu \nu}^{M} & =\xi_{\mu \nu}^{M}-\delta_{\mu}^{M, T} d^{-1} \delta_{\nu}^{M}-\vartheta_{\mu}^{M, T} \Theta^{-1} \vartheta_{\nu}^{M}, M=\mathcal{Z}, \mathcal{A}, \\
\widetilde{\eta}_{\mu \nu}^{\mathcal{A}} & =\eta_{\mu \nu}^{\mathcal{A}}-\delta_{\mu}^{\mathcal{A}, T} d^{-1} \delta_{\nu}^{\mathcal{A}}-\vartheta_{\mu}^{\mathcal{A}, T} \Theta^{-1} \vartheta_{\nu}^{\mathcal{A}} .
\end{aligned}
$$

Similar relations hold for the transposed components $\widetilde{\xi}_{\mu \nu}^{\mathcal{Z}, T}, \widetilde{\xi}_{\mu \nu}^{\mathcal{A}, T}$, and $\widetilde{\eta}_{\mu \nu}^{\mathcal{A}, T}$. Furthermore, we have introduced the quantities

$$
\vartheta_{\mu}^{M}=\Delta_{\mu}^{M}-\delta^{T} d^{-1} \delta_{\mu}^{M}, \quad M=\mathcal{W}, \mathcal{Z}, \mathcal{A}
$$

Note that the index $\mathcal{W}$ which appears in Eqs. (4.38), (4.39), and (4.42) refers to the two components $w_{\mu}^{1,2}$ of the fluctuations which correspond to the original fields $W_{\mu}^{1,2}$. We will use Greek letters $\alpha, \beta=1,2$ to label these two components. The expressions for the differential operators which appear in Eqs. (4.39)-(4.41) can be found in Appendix A. The operators $\delta_{\mu}$ and $\Delta_{\mu}$ are listed in Eqs. (A24)-A35. The first terms on the right-hand side of Eqs. (4.39)-(4.41) are given by Eqs. (A36)-(A44).

Finally, we can diagonalize the differential operator $\widetilde{\mathcal{D}}_{\mu \nu}$ from Eq. (4.38) in an analogous way to the diagonalization of the operator $\underline{\widetilde{D}}+\underline{P P^{T}}+\delta_{P}$ in Eq. (4.33). The corresponding transformation matrix has again unit determinant. This is, of course, also true for the orthogonal matrix $O$, Eq. (4.37). Therefore we can write the generating functional in the following form:

$$
W_{\mathrm{SM}}\left[h, K_{\mu \nu}, J_{\mu}^{a}\right]=\int \mathrm{d}^{d} x \mathcal{L}_{\mathrm{SM}}+\frac{1}{2} \ln \operatorname{det} d+\frac{1}{2} \ln \operatorname{det} \Theta+\frac{1}{2} \ln \operatorname{det} \mathcal{D}^{\mathcal{W}}+\frac{1}{2} \ln \operatorname{det} \mathcal{D}^{\mathcal{Z}}+\frac{1}{2} \ln \operatorname{det} \mathcal{D}^{\mathcal{A}}-\ln \operatorname{det} \underline{P^{T} P} .
$$

The operators which appear in Eq. (4.43) are defined through the relations

$$
\begin{aligned}
\mathcal{D}_{\mu \nu}^{\mathcal{W}} & \equiv \widetilde{D}_{\mu \nu}^{\mathcal{W}}=D_{\mu \nu}^{\mathcal{W}}-\delta_{\mu}^{\mathcal{W}, T} d^{-1} \delta_{\nu}^{\mathcal{W}}-\vartheta_{\mu}^{\mathcal{W}, T} \Theta^{-1} \vartheta_{\nu}^{\mathcal{W}}, \\
\mathcal{D}_{\mu \nu}^{\mathcal{Z}} & =\widetilde{D}_{\mu \nu}^{\mathcal{Z}}-\widetilde{\xi}_{\mu \rho}^{\mathcal{Z}, T}\left(\mathcal{D}^{\mathcal{W}}\right)_{\rho \sigma}^{-1} \widetilde{\xi}_{\sigma \nu}^{\mathcal{Z}}, \\
\mathcal{D}_{\mu \nu}^{\mathcal{A}} & =\widetilde{D}_{\mu \nu}^{\mathcal{A}}-\widetilde{\xi}_{\mu \rho}^{\mathcal{A}, T}\left(\mathcal{D}^{\mathcal{W}}\right)_{\rho \sigma}^{-1} \widetilde{\xi}_{\sigma \nu}^{\mathcal{A}}-N_{\mu \rho}^{\mathcal{A}, T}\left(\mathcal{D}^{\mathcal{Z}}\right)_{\rho \sigma}^{-1} N_{\sigma \nu}^{\mathcal{A}}, \\
N_{\mu \nu}^{\mathcal{A}} & =\widetilde{\eta}_{\mu \nu}^{\mathcal{A}}-\widetilde{\xi}_{\mu \rho}^{\mathcal{Z}, T}\left(\mathcal{D}^{\mathcal{W}}\right)_{\rho \sigma}^{-1} \widetilde{\xi}_{\sigma \nu}^{\mathcal{A}} .
\end{aligned}
$$

Equation (4.28) and the equivalent forms in Eqs. (4.35) and (4.43) represent our result for the generating functional $W_{\mathrm{SM}}\left[h, K_{\mu \nu}, J_{\mu}^{a}\right]$ for the gauge-invariant Green's functions for the bosonic sector of the standard model. These formulas encode the full tree-level and one-loop effects of the theory. If one expands the generating functional up to a given order in powers of the external sources one can extract any $n$-point Green's functions for the gauge-invariant operators $\Phi^{\dagger} \Phi, B_{\mu \nu}$, and $\underline{V}_{\mu}^{a}$. Since the equations of motion (3.2)-(3.7) only involve gauge-invariant fields and because the differential operators which enter the generating functional only contain gauge-invariant quantities, cf. the explicit expressions in Appendix A, the evaluation of the generating functional and the final result will be manifestly gaugeinvariant. In Sec. VI we will calculate the two-point functions of these gauge-invariant operators. Before we come to this, we discuss in the next section the renormalization of the theory in the presence of the external sources.

\section{RENORMALIZATION}

In order to render the generating functional $W_{\mathrm{SM}}\left[h, K_{\mu \nu}, J_{\mu}^{a}\right]$ of the standard model in Eq. (4.28), and the equivalent forms in Eqs. (4.35) and (4.43), finite, one has to renormalize the bare constants $m^{2}, \lambda, g, g^{\prime}$, the scalar field $\Phi$ and the sources before the regulator can be removed. There is no wave-function renormalization for the gauge fields $W_{\mu}^{a}$ and $B_{\mu}$ on account of gauge invariance, cf. our definition of the covariant derivative in Eq. (2.2). The ultraviolet divergences are related to the poles of the $d$-dimensional determinant, which appear in the generating functional for $d=0,2,4, \ldots$. In general, for a differential operator $\bar{D}$ of the form

$$
\bar{D}=-\bar{D}_{\mu} \bar{D}_{\mu}+\bar{\sigma}, \quad \bar{D}_{\mu}=\partial_{\mu}+\bar{\Gamma}_{\mu},
$$

the pole term of the determinant at $d=4$ is given by 


$$
\frac{1}{2} \ln \operatorname{det} \bar{D}=\frac{1}{d-4} \frac{1}{16 \pi^{2}} \int \mathrm{d}^{d} x \operatorname{tr}\left(\frac{1}{12} \bar{\Gamma}_{\mu \nu} \bar{\Gamma}_{\mu \nu}+\frac{1}{2} \bar{\sigma}^{2}\right)+\mathcal{O}(1)
$$

with

$$
\bar{\Gamma}_{\mu \nu}=\left[\bar{D}_{\mu}, \bar{D}_{\nu}\right] .
$$

This identity can readily be derived [18] using the heat kernel method [30]. The symbol "tr" in Eq. (5.2) denotes the trace over internal and Lorentz indices which will be suppressed in the following. We cannot apply, however, formula (5.2) in our case. This is due to the fact that the differential operator ${ }^{3} \widetilde{D}+\bar{P} \bar{P}^{T}$, which appears in the one-loop expression for the generating functional, is not an ordinary local differential operator of the form given in Eq. (5.1), but a nonlocal functional of the fields due to the presence of derivatives of the massless propagator $\mathcal{G}_{0}(x-y)=\langle x|1 /(-\square)| y\rangle$ in some of the components. These massless propagators originate from the phase factor $\varphi$, cf. Eq. (2.22). In order to calculate the counterterms, we therefore split the differential operator in a local, $D_{\text {loc }}$, and a "nonlocal," $\delta D$, part

$$
\widetilde{D}+\bar{P} \bar{P}^{T}=D_{\text {loc }}+\delta D,
$$

where $\delta D$ contains all the terms stemming from the phase factor. The explicit expressions for these operators can be found in Appendix B, cf. Eqs. (B10) and (B21). Using this decomposition we can write

$$
\frac{1}{2} \ln \operatorname{det}\left(\widetilde{D}+\bar{P} \bar{P}^{T}\right)=\frac{1}{2} \ln \operatorname{det} D_{\text {loc }}+\frac{1}{2} \operatorname{Tr}\left[D_{\text {loc }}^{-1} \delta D\right]-\frac{1}{4} \operatorname{Tr}\left[\left(D_{\text {loc }}^{-1} \delta D\right)^{2}\right]+\frac{1}{6} \operatorname{Tr}\left[\left(D_{\text {loc }}^{-1} \delta D\right)^{3}\right]+\cdots .
$$

The first term on the right-hand side of Eq. (5.5) can now be treated in the usual way using Eq (5.2), whereas we can use a short distance expansion to extract the divergent and local contributions from the other terms. Techniques for performing such a short distance expansion have been discussed in detail in Ref. [18]. Here we present only the general procedure.

We write the second term on the right-hand side of Eq. (5.5) in the form

$$
\frac{1}{2} \operatorname{Tr}\left[D_{\text {loc }}^{-1} \delta D\right]=\frac{1}{2} \int \mathrm{d}^{d} x \mathrm{~d}^{d} y \operatorname{tr}\left(\left\langle x\left|D_{\text {loc }}^{-1}\right| y\right\rangle\langle y|\delta D| x\rangle\right) .
$$

Due to the presence of one propagator $D_{\text {loc }}^{-1}$ this term can be viewed as a tadpole graph.

The ultraviolet poles in Eq. (5.6) are connected to the short distance properties of the operator $D_{\text {loc }}$, which in turn are governed by the Laplacian $\square$, since $D_{\text {loc }}$ is of the form given in Eq. (5.1). Observing that in $d$-dimensional Euclidean space one has the identity

$$
\left\langle x\left|e^{\lambda \square}\right| y\right\rangle=(4 \pi \lambda)^{-d / 2} e^{-z^{2} / 4 \lambda},
$$

where $z=x-y$, we define the heat kernel $H(x|\lambda| y)$ through the equation

$$
\left\langle x\left|e^{-\lambda D_{\mathrm{loc}}}\right| y\right\rangle \doteq(4 \pi \lambda)^{-d / 2} e^{-z^{2} / 4 \lambda} H(x|\lambda| y) .
$$

Using Eq. (5.8) we can then write

$$
\begin{aligned}
\left\langle x\left|D_{\mathrm{loc}}^{-1}\right| y\right\rangle & =\int_{0}^{\infty} \frac{\mathrm{d} \lambda}{(4 \pi \lambda)^{d / 2}} e^{-z^{2} / 4 \lambda} H(x|\lambda| y) \\
& =\sum_{n=0}^{\infty} \int_{0}^{\infty} \frac{\mathrm{d} \lambda}{(4 \pi \lambda)^{d / 2}} e^{-z^{2} / 4 \lambda} \lambda^{n} H_{n}(x \mid y) .
\end{aligned}
$$

In the second line we have expanded the heat kernel $H(x|\lambda| y)$ in terms of the heat coefficients $H_{n}(x \mid y)$. These heat coefficients can in turn be expanded around the point $x$ in order to obtain local counterterms at the end:

\footnotetext{
${ }^{3}$ For the calculation of the counterterms it is convenient to introduce a real representation for the Higgs field. See Appendix B for details.
} 


$$
H_{n}(x \mid y)=H_{n}(x \mid x)-\left.z_{\mu}\left(\partial_{\mu} H_{n}\right)\right|_{x=y}+\left.\frac{1}{2} z_{\mu} z_{\nu}\left(\partial_{\mu} \partial_{\nu} H_{n}\right)\right|_{x=y}+\cdots .
$$

One now inserts the resulting expression for $\left\langle x\left|D_{\text {loc }}^{-1}\right| y\right\rangle$ into Eq. (5.6). Moreover, one can use the following representations for the massless propagator $\mathcal{G}_{0}$ and derivatives thereof

$$
\begin{aligned}
\mathcal{G}_{0}(z) & =\int_{0}^{\infty} \frac{\mathrm{d} \rho}{(4 \pi \rho)^{d / 2}} e^{-z^{2} / 4 \rho} \\
\partial_{\mu} \mathcal{G}_{0}(z) & =\int_{0}^{\infty} \frac{\mathrm{d} \rho}{(4 \pi \rho)^{d / 2}}\left(-\frac{1}{2} z_{\mu} \frac{1}{\rho}\right) e^{-z^{2} / 4 \rho} \\
\partial_{\mu} \partial_{\nu} \mathcal{G}_{0}(z) & =\int_{0}^{\infty} \frac{\mathrm{d} \rho}{(4 \pi \rho)^{d / 2}}\left(-\frac{1}{2} \delta_{\mu \nu} \frac{1}{\rho}+\frac{1}{4} z_{\mu} z_{\nu} \frac{1}{\rho^{2}}\right) e^{-z^{2} / 4 \rho},
\end{aligned}
$$

which appear in $\langle y|\delta D| x\rangle$. If one now performs the integration over $d^{d} z$ and the parameters $\rho$ and $\lambda$, one observes that the ultraviolet poles manifest themselves as divergences at the lower end of the integration over $\lambda$. Power counting shows that higher order heat coefficients $H_{n}$ lead to a less singular behavior for short distances since they are accompanied by additional powers of $\lambda$ in Eq. (5.10). The same is true for higher derivative terms in the expansion of $H_{n}$ in Eq. (5.11), due to the presence of additional powers of $z_{\mu}$. Therefore only a finite number of terms in the expansions in Eqs. (5.10) and (5.11) lead to ultraviolet divergent contributions. At the end, we need only the leading order term $H_{0}(x \mid x)$ and the next-to-leading order terms $\left.\left(\partial_{\mu} \partial_{\nu} H_{0}\right)\right|_{x=y}$ and $H_{1}(x \mid x)$ to extract the counterterms. The explicit expressions for these quantities can be inferred from the results (cf. Ref. [18] for the derivation):

$$
\begin{aligned}
H_{0}(x \mid x) & =1, \quad H_{1}(x \mid x)=-\bar{\sigma}, \\
\left.\left(\bar{D}_{\mu} H_{0}\right)\right|_{x=y} & =0,\left.\quad\left(\bar{D}_{\mu} \bar{D}_{\nu} H_{0}\right)\right|_{x=y}=\frac{1}{2} \bar{\Gamma}_{\mu \nu} .
\end{aligned}
$$

The third term in Eq. (5.5) can be written in the form

$$
-\frac{1}{4} \operatorname{Tr}\left[\left(D_{\mathrm{loc}}^{-1} \delta D\right)^{2}\right]=-\frac{1}{4} \int \mathrm{d}^{d} x \mathrm{~d}^{d} y \mathrm{~d}^{d} z \mathrm{~d}^{d} u \operatorname{tr}\left(\left\langle x\left|D_{\mathrm{loc}}^{-1}\right| y\right\rangle\langle y|\delta D| z\rangle\left\langle z\left|D_{\mathrm{loc}}^{-1}\right| u\right\rangle\langle u|\delta D| x\rangle\right),
$$

which can be interpreted as a two-point function with two propagators $D_{\text {loc }}^{-1}$. Similar arguments as used above then lead to the observation that we need only the leading term in the short distance behavior of $D_{\text {loc }}$, which amounts to setting

$$
\left\langle x\left|D_{\mathrm{loc}}^{-1}\right| y\right\rangle \rightarrow\left\langle x\left|\frac{1}{-\square}\right| y\right\rangle,
$$

in Eq. (5.16). Again we have suppressed all internal and Lorentz indices in Eq. (5.17). Finally, all the subsequent terms in Eq. (5.5), which contain three and more propagators $D_{\text {loc }}^{-1}$, lead to ultraviolet finite integrals.

The ultraviolet contributions from the measure of the path integral to the generating functional, i.e. the term $-\ln \operatorname{det} \bar{P}^{T} \bar{P}$, can be treated in the usual way, using Eq. (5.2). The explicit result for the operator $\bar{P}^{T} \bar{P}$ can be found in Eq. (B25).

This procedure leads to the counterterm Lagrangian from which one can read off the renormalization prescriptions which will remove the poles in the generating functional in Eq. (4.28). The full list of renormalization prescriptions for all the fields, the mass parameter $m^{2}$, the coupling constants, and the sources are listed in Appendix B in Eqs. (B26)$(\mathrm{B} 33)$, and $(\mathrm{B} 35)-(\mathrm{B} 60)$. In the next section we will only need the renormalization prescriptions for the fields, the mass and the coupling constants which are given in Eqs. (B26) $-($ B33).

\section{PHYSICAL INPUT PARAMETERS FROM GAUGE-INVARIANT GREEN'S FUNCTIONS}

In this section we relate the bare parameters of the theory to physical quantities. As physical input parameters we choose the masses of the Higgs and the $W$ and $Z$ bosons, and the electric charge (on-shell scheme). The physical mass of the Higgs boson, which we denote by $M_{H, \text { pole }}^{2}$, is determined by the pole position of the two-point function

$$
\left\langle 0\left|T\left(\Phi^{\dagger} \Phi\right)(x)\left(\Phi^{\dagger} \Phi\right)(y)\right| 0\right\rangle .
$$


The physical masses of the $W$-boson, $M_{W \text {,pole }}^{2}$, and the $Z$-boson, $M_{Z, \text { pole }}^{2}$, are defined by the pole positions of the two-point function

$$
\left\langle 0\left|T\left(\underline{V}_{\mu}^{a}\right)(x)\left(\underline{V}_{\nu}^{b}\right)(y)\right| 0\right\rangle .
$$

The electric charge is determined by the three-point function

$$
\left\langle 0\left|T B_{\mu \nu}(x)\left(\underline{V}_{\rho}^{+}\right)(y)\left(\underline{V}_{\sigma}^{-}\right)(z)\right| 0\right\rangle .
$$

Alternatively, one can define a renormalized electric charge as the residue at the photon pole of the two-point function

$$
\left\langle 0\left|T B_{\mu \nu}(x) B_{\rho \sigma}(y)\right| 0\right\rangle \text {. }
$$

We will denote the corresponding coupling constant by $e_{\text {res }}^{2}$. At this point several comments are in order. In the usual approach to gauge theories dealing with gauge-dependent Green's functions, two- and three-point functions are related by Ward identities. These kind of Ward identities follow, however, from the fact that these Green's functions are gauge-dependent. In contrast, there are no Ward identities of this type between our gauge-invariant Green's functions. Nevertheless, the absence of Ward identities does not imply any lack of knowledge. All information that Ward identities encode in any gauge-dependent framework is manifest in our approach. Therefore, one may expect the coupling constant which can be extracted from the three-point function in Eq. (6.3) and from the two-point function in Eq. (6.4) to be the same, if they are evaluated at the same scale. Below, we will extract the electric charge $e_{\text {res }}^{2}$ from the residue of the two-point function in Eq. (6.4). We have not explicitly checked whether the definition through the three-point function in Eq. (6.3) leads to the same result. Note that the residue of the two-point function of the field strength $B_{\mu \nu}$ in Eq. (6.4) differs from unity and that there is no freedom to adjust the residue by a renormalization factor. This can be traced back to our normalization of the gauge field $B_{\mu}$ in the covariant derivative, cf. Eq. (2.2).

Before we begin with the evaluation of the physical input parameters let us discuss an example for relations that usually are derived with the help of Ward identities, but which are manifest in our approach. Consider the covariant derivative $D_{\mu}$ as defined in Eq. (2.2). Gauge invariance ensures that the covariant structure of $D_{\mu}$ is not destroyed by counterterms. In fact all counterterms are gauge-invariant in our approach. Therefore the fields $W_{\mu}^{a}$ and $B_{\mu}$ are not renormalized, cf. Eqs. (B26) and (B27). Factoring out the gauge coupling constants $g$ and $g^{\prime}$ from the gauge fields, which we then denote by $\hat{W}_{\mu}^{a}$ and $\hat{B}_{\mu}$, the covariant derivative reads

$$
D_{\mu} \Phi=\left(\partial_{\mu}-i \frac{\tau^{a}}{2} g \hat{W}_{\mu}^{a}-i \frac{1}{2} g^{\prime} \hat{B}_{\mu}\right) \Phi .
$$

Thus, the wave function renormalization of the field $\hat{W}_{\mu}^{a}\left(\hat{B}_{\mu}\right)$ must be the inverse of the renormalization for $g\left(g^{\prime}\right)$. This follows automatically from gauge invariance.

For the determination of the two-point functions in Eqs. (6.1), (6.2), and (6.4) we need the generating functional $W_{\mathrm{SM}}\left[h, K_{\mu \nu}, J_{\mu}^{a}\right]$ up to second order in the external sources. Using a saddle-point approximation for the path integral, the generating functional at tree level is given by the action, evaluated at the solutions of the classical equations of motion. Inserting the solutions of the equation of motion from Eqs. (3.17) $-(3.22)$ into the classical action we get the following result for the generating functional at tree level, expanded up to second order in powers of the external sources:

$$
\begin{aligned}
W_{\mathrm{SM}}\left[h, K_{\mu \nu}, J_{\mu}^{a}\right]^{\text {tree }}= & \int d^{d} x\left\{-\frac{m^{2}}{2 \lambda} h(x)\right\}+\int d^{d} x d^{d} y\left\{-\frac{m^{2}}{2 \lambda} h_{x} \mathcal{G}_{H}(x-y) h_{y}-\frac{8 m^{2}}{\lambda} M_{W}^{2}\left(J_{\mu, x}^{+, T} \mathcal{G}_{W}(x-y) J_{\mu, y}^{-, T}\right)\right. \\
& -\frac{s^{2} c^{2}}{2 e^{2}}\left(\frac{e^{2}}{c^{2}}\left(\partial_{\nu} \widehat{K}_{\nu \mu}\right)-4 M_{Z}^{2} J_{\mu}^{\mathcal{Z}, T}\right)_{x} \mathcal{G}_{Z}(x-y)\left(\frac{e^{2}}{c^{2}}\left(\partial_{\rho} \widehat{K}_{\rho \mu}\right)-4 M_{Z}^{2} J_{\mu}^{\mathcal{Z}, T}\right)_{y} \\
& \left.-\frac{1}{2} e^{2}\left(\partial_{\nu} \widehat{K}_{\nu \mu}\right)_{x} \mathcal{G}_{A}(x-y)\left(\partial_{\rho} \widehat{K}_{\rho \mu}\right)_{y}+\text { contact terms }\right\}
\end{aligned}
$$

We have indicated the space-time arguments of the sources by the subscripts $x$ and $y$.

The contact terms in Eq. (6.6) do not contribute to the pole positions of the two-point functions. Note that only the transversal modes are propagating at this order of the expansion. From the pole positions of the propagators we can read off the masses of the particles at tree level:

$$
M_{H}^{2}=2 m^{2} ; M_{W}^{2} ; M_{Z}^{2} ; M_{\gamma}^{2}=0 .
$$


The two-point function of the field strength $B_{\mu \nu}$ in Eq. (6.4) is obtained from the generating functional in Eq. (6.6), if we switch off all the sources, except for $K_{\mu \nu}$. It contains poles at $p^{2}=0$ and at $p^{2}=-M_{Z}^{2}$ (note that we are working in Euclidean space-time), because of the presence of the propagators $\mathcal{G}_{A}$ and $\mathcal{G}_{Z}$ in Eq. (6.6). This is due to the fact that the field $B_{\mu}$ is a linear combination of the photon and the $Z$-boson field, cf. Eq. (2.15). From the residue at the pole position of the photon we get the electric charge

$$
e_{\mathrm{res}}^{2}=e^{2} .
$$

At the one-loop level the generating functional is given by the expression in Eq. (4.43), where the diagonalization of the differential operator for the quantum fluctuations has been carried out completely. Expanding the determinants in powers of the external sources leads after a lengthy calculation to the following result for the generating functional:

$$
\begin{aligned}
W_{\mathrm{SM}}\left[h, K_{\mu \nu}, J_{\mu}^{a}\right]= & \int \frac{d^{d} p}{(2 \pi)^{d}}\left\{-\frac{m^{2}}{2 \lambda} h(p)-\left(\frac{m^{2}}{2 \lambda}\right) h(p) \mathcal{G}_{H}(p)\left[1+\Sigma_{\Phi^{\dagger} \Phi}\left(p^{2}\right) \mathcal{G}_{H}(p)\right] h(-p)\right. \\
& -\left(\frac{8 m^{2}}{\lambda} M_{W}^{2}\right) J_{\mu}^{+, T}(p) \mathcal{G}_{W}(p)\left[1+\Sigma_{\underline{\mathcal{W}}}^{T}\left(p^{2}\right) \mathcal{G}_{W}(p)\right] J_{\mu}^{-, T}(-p) \\
& -\frac{1}{2}\left(\widetilde{J}_{\mu}^{\mathcal{Z}, T}(p), \widetilde{J}_{\mu}^{\mathcal{A}, T}(p)\right) \mathcal{G}_{Z \gamma}(p)\left[\mathbf{1}+\boldsymbol{\Sigma}_{\mathcal{Z} \gamma}^{T}\left(p^{2}\right) \mathcal{G}_{Z \gamma}(p)\right]\left(\begin{array}{c}
\widetilde{J}_{\mu}^{\mathcal{Z}, T}(-p) \\
\widetilde{J}_{\mu}^{\mathcal{A}, T}(-p)
\end{array}\right) \\
& \left.+16 J_{\mu}^{+, L}(p) \Sigma_{\underline{\mathcal{W}}}^{L}\left(p^{2}\right) J_{\mu}^{-, L}(-p)+4 J_{\mu}^{\mathcal{Z}, L}(p) \Sigma_{\mathcal{Z}}^{L}\left(p^{2}\right) J_{\mu}^{\mathcal{Z}, L}(-p)+\text { contact terms }\right\},
\end{aligned}
$$

where we have introduced the abbreviations

$$
\begin{aligned}
\widetilde{J}_{\mu}^{\mathcal{Z}, T}(p) & =\frac{s c}{e}\left(-4 M_{Z}^{2} J_{\mu}^{\mathcal{Z}, T}(p)+\frac{e^{2}}{c^{2}}\left(\partial_{\rho} \widehat{K}_{\rho \mu}(p)\right)\right), \\
\widetilde{J}_{\mu}^{\mathcal{A}, T}(p) & =e\left(\partial_{\rho} \widehat{K}_{\rho \mu}(p)\right), \\
\mathcal{G}_{Z \gamma}(p) & =\left(\begin{array}{cc}
\mathcal{G}_{Z}(p) & 0 \\
0 & \mathcal{G}_{A}(p)
\end{array}\right), \\
\boldsymbol{\Sigma}_{\mathcal{Z} \gamma}^{T}\left(p^{2}\right) & =\left(\begin{array}{cc}
\Sigma_{\mathcal{Z}}^{T}\left(p^{2}\right) & \Sigma_{\mathcal{Z}}^{T}\left(p^{2}\right) \\
\Sigma_{\mathcal{A Z}}^{T}\left(p^{2}\right) & \Sigma_{\mathcal{A}}^{T}\left(p^{2}\right)
\end{array}\right), \\
\mathbf{1} & =\left(\begin{array}{cc}
1 & 0 \\
0 & 1
\end{array}\right) .
\end{aligned}
$$

The propagators $\mathcal{G}$ in Eq. (6.9) are defined in Eq. (3.24). The explicit results for the self-energies $\Sigma$ can be found in Appendix G. We note that these self-energies are part of our gauge-invariant Green's functions and should not be confused with the self-energies of the Higgs boson and the gauge fields in the usual approach.

Since our calculation preserves gauge invariance, the self-energy $\Sigma_{\mathcal{A}}^{T}$ from Eq. (C9) has the property $\Sigma_{\mathcal{A}}^{T}(0)=0$ which guarantees that the photon remains massless. From the result in Eq. (C6) follows that $\Sigma_{\mathcal{Z} \mathcal{A}}^{T}(0)=0$. Therefore the self-energy mixing-matrix $\boldsymbol{\Sigma}_{\mathcal{Z} \gamma}^{T}\left(p^{2}\right)$ in Eq. (6.13) is diagonal at $p^{2}=0$. Furthermore, gauge invariance implies that the equations of motion and the differential operators only contain the transverse component $\mathcal{A}_{\mu}^{T}$. Therefore only the self-energies $\Sigma_{\mathcal{Z} \mathcal{A}}^{T}$ and $\Sigma_{\mathcal{A}}^{T}$ appear in the generating functional in Eq. (6.9). There are no quantities $\Sigma_{\mathcal{Z} \mathcal{A}}^{L}$ and $\Sigma_{\mathcal{A}}^{L}$. All these properties of the self-energies follow directly from gauge invariance. There is no need to impose them by any kind of renormalization conditions. We note that these properties for the self-energies also hold for the corresponding self-energies of the gauge fields in the background field approach to the standard model [16]. The latter Green's functions still depend, however, on the gauge fixing parameter $\xi_{Q}$ for the quantum fluctuations.

As a check on our calculation we have verified that in the limit $g^{\prime} \rightarrow 0$ the self-energies $\Sigma_{\mathcal{Z}}$ and $\Sigma_{\mathcal{W}}$ coincide. This statement is true for the transversal self-energies, $\Sigma^{T}$, Eqs. (C2) and (C4), as well as the longitudinal self-energies $\Sigma^{L}$, Eqs. (C3) and (C5).

From the expression for the generating functional in Eq. (6.9) we define the full propagators:

$$
\begin{aligned}
\mathcal{G}^{\text {full }}\left(p^{2}\right) & =\mathcal{G}\left(p^{2}\right)\left(1+\Sigma\left(p^{2}\right) \mathcal{G}\left(p^{2}\right)\right) \\
& =\frac{1}{p^{2}+M^{2}-\Sigma\left(p^{2}\right)}
\end{aligned}
$$


The second line follows after Dyson resummation. In Eq. (6.16) we denoted the bare mass by $M^{2}$. For the gauge bosons we consider only the transverse components. The definition in Eq. (6.15) can be applied to the mixing propagator $\mathcal{G}_{Z \gamma}^{\text {full }}$, as well.

Using the explicit results from Appendix $\mathrm{O}$ one observes that all self-energies behave for large momenta as

$$
\Sigma\left(p^{2}\right) \sim p^{2} \ln \left(p^{2} / \mu^{2}\right) \text { for } p^{2} \rightarrow \infty .
$$

Note that there are individual contributions to the self-energies which grow like $p^{4}$. They cancel each other, however, in the large $p^{2}$ limit. Therefore, the full propagators in Eq. (6.15) or (6.16) have the proper high energy behavior proportional to $1 /\left(p^{2} \ln \left(p^{2} / \mu^{2}\right)\right)$. This can be traced back to the fact that the Goldstone boson modes are present in the calculation of the generating functional at one-loop level. The propagators in Eq. (6.15) or (6.16) are not identical to the propagators in the unitary gauge in the usually employed formalism.

We note that there are terms proportional to $1 / p^{2}$ in $\Sigma_{\underline{\mathcal{W}}}^{T, L}$ and $\Sigma_{\mathcal{Z}}^{T, L}$, cf. Eqs. (C2)-( $\mathrm{C} 5$ ). The limit $p^{2} \rightarrow 0$ in these self-energies is, however, well defined.

We define the physical masses of the particles through the pole position of the two-point functions in Eqs. (6.1) and (6.2). These poles then appear in the full, resummed propagators as defined in Eq. (6.16). The masses of the $Z$-boson and the photon can be identified with the eigenvalues of the inverse full mixing-propagator, i.e. the zeros of the determinant of $\left(\mathcal{G}_{Z \gamma}^{\text {full }}\right)^{-1}$. At the one-loop level we get the relations

$$
\begin{aligned}
M_{H, \text { pole }}^{2} & \doteq 2 m^{2}-\operatorname{Re}\left[\Sigma_{\Phi^{\dagger} \Phi}\left(\mathrm{p}^{2}=-\mathrm{M}_{\mathrm{H}, \mathrm{pole}}^{2}\right)\right] \\
& \approx 2 m^{2}-\operatorname{Re}\left[\Sigma_{\Phi^{\dagger} \Phi}\left(\mathrm{p}^{2}=-2 \mathrm{~m}^{2}\right)\right], \\
M_{W, \text { pole }}^{2} & \doteq M_{W}^{2}-\operatorname{Re}\left[\Sigma_{\underline{\mathcal{W}}}^{\mathrm{T}}\left(\mathrm{p}^{2}=-\mathrm{M}_{\mathrm{W}, \mathrm{pole}}^{2}\right)\right] \\
& \approx M_{W}^{2}-\operatorname{Re}\left[\Sigma_{\underline{\mathcal{W}}}^{\mathrm{T}}\left(\mathrm{p}^{2}=-\mathrm{M}_{\mathrm{W}}^{2}\right)\right], \\
M_{Z, \text { pole }}^{2} & \doteq M_{Z}^{2}-\operatorname{Re}\left[\Sigma_{\mathcal{Z}}^{\mathrm{T}}\left(\mathrm{p}^{2}=-\mathrm{M}_{\mathrm{Z}, \text { pole }}^{2}\right)\right] \\
& \approx M_{Z}^{2}-\operatorname{Re}\left[\Sigma_{\mathcal{Z}}^{\mathrm{T}}\left(\mathrm{p}^{2}=-\mathrm{M}_{\mathrm{Z}}^{2}\right)\right] .
\end{aligned}
$$

The approximations are valid at the one-loop level. Note that we are working in Euclidean space-time. Furthermore, only bare quantities enter on the right-hand side of the Eqs. (6.19), (6.21), and (6.23). The photon remains massless due to the relation $\Sigma_{\mathcal{A}}^{T}(0)=0$. At the one-loop level only the diagonal elements of the self-energy $\boldsymbol{\Sigma}_{\mathcal{Z} \gamma}^{T}$ from Eq. (6.13) enter the definitions for the physical masses of $Z$-boson and the photon. The explicit expressions for the physical masses can be inferred from the results for the self-energies given in Appendix $\mathrm{C}$. We do not list them here because they are too lengthy.

We define the electric charge $e_{\text {res }}^{2}$ as the residue at the photon pole of the two-point function $\left\langle 0\left|T B_{\mu \nu}(x) B_{\rho \sigma}(y)\right| 0\right\rangle$. As discussed before, this two-point function has poles at $p^{2}=0$ and at $p^{2}=-M_{Z}^{2}$. Due to the fact that $\Sigma_{\mathcal{Z} \mathcal{A}}^{T}(0)=0$, the residue at the photon pole is given by the expression

$$
e_{\mathrm{res}}^{2} \doteq \frac{e^{2}}{1-\left.\frac{\partial}{\partial p^{2}} \Sigma_{\mathcal{A}}^{T}\left(p^{2}\right)\right|_{p^{2}=0}} \approx e^{2}\left(1+\left.\frac{\partial}{\partial p^{2}} \Sigma_{\mathcal{A}}^{T}\left(p^{2}\right)\right|_{p^{2}=0}\right) .
$$

The approximation used is valid at the one-loop level. From the expression for $\Sigma_{\mathcal{A}}^{T}$ in Eq. (C9) we get the following relation between the physical coupling constant $e_{\text {res }}^{2}$ and the bare coupling constant $e^{2}$ :

$$
\begin{aligned}
& e_{\mathrm{res}}^{2}=e^{2}\left(1+e^{2} \delta e^{2}\right), \\
& \delta e^{2}=-14\left[\Lambda_{\varepsilon}\left(2 m^{2}\right)+\frac{1}{32 \pi^{2}} \ln \left(\frac{M_{W}^{2}}{2 m^{2}}\right)\right]-\frac{19}{3} \frac{1}{16 \pi^{2}},
\end{aligned}
$$

where

$$
\Lambda_{\varepsilon}\left(2 m^{2}\right) \doteq \frac{\mu^{d-4}}{16 \pi^{2}}\left(\frac{1}{d-4}-\frac{1}{2}\left(\ln (4 \pi)+\Gamma^{\prime}(1)+1\right)\right)+\frac{1}{32 \pi^{2}} \ln \left(\frac{2 m^{2}}{\mu^{2}}\right) .
$$

Only bare quantities appear on the right-hand side of Eq. (6.25). We note that the result for $e_{\text {res }}^{2}$ agrees with the usual definition of the electric charge in the Thompson limit [31] in the absence of fermion contributions.

The expressions for the physical masses, Eqs. (6.19), (6.21), (6.23), and the electric charge $e_{\mathrm{res}}^{2}$, Eq. (6.25), are finite if one inserts the renormalization prescriptions from Eqs. (B26)-(B33) for the bare quantities. The cancellation of the pole terms served as an important test of our calculation. 


\section{SUMMARY AND DISCUSSION}

In this article we have presented a manifestly gauge-invariant approach to the bosonic sector of the standard model. Its essential feature is to consider gauge-invariant Green's functions. Hence, the generating functional involves external sources that couple to gauge-invariant operators only. In order to obtain the same $S$-matrix elements as in the usual gauge-dependent approach, we chose sources that emit one-particle states of the Higgs boson, the $W$ - and the $Z$-boson and the photon. In addition to that, however, the off-shell behavior of our Green's functions is completely free of any gauge-artifacts. This property makes our approach particularly suitable for situations where one is interested to gain information from off-shell quantities or where one is forced to deal with them, like, for instance, the analysis of finite width effects or the parametrization of new physics in terms of the oblique parameters $S, T$ and $U$.

As gauge-invariant operators we chose the scalar density $\Phi^{\dagger} \Phi$, the Abelian field strength $B_{\mu \nu}$ and the quantities $\varphi^{a b} V_{\mu}^{b}$, where $V_{\mu}^{b}$ are the currents of the global $S U(2)_{R}$ symmetry, cf. Eqs. (2.11) and (2.33). The third component of the current, $V_{\mu}^{3}$, the scalar density and the Abelian field strength are already invariant under the full gauge-group $S U(2)_{L} \times U(1)_{Y}$. The other two components of the current are only invariant under the non-Abelian subgroup $S U(2)_{L}$ but transform non-trivially under the Abelian group. In order to ensure full $S U(2)_{L} \times U(1)_{Y}$ invariance, we introduced an Abelian phase factor $\varphi^{a b}$ coupling to the charged $S U(2)_{R}$ currents as given in Eq. (2.33). The scalar density and the Abelian field strength excite one-particle states of the Higgs boson and the photon, respectively, while the currents emit one-particle states of the massive gauge fields.

Since we couple external sources to gauge-invariant operators only, the generating functional can be defined in terms of a path integral without the need to fix a gauge. At tree level, it is given by the classical action. The equations of motion determine only the physical degrees of freedom. Hence, they have a whole class of solutions in terms of the original fields. Every two representatives are related to each other by a gauge transformation. Since the action is gauge-invariant, the generating functional is uniquely determined. An important property of our approach is the fact, that the classical Goldstone boson field represents the $S U(2)_{L}$ gauge degrees of freedom. Thus, no Goldstone boson fields are propagating at the classical level of the theory. All gauge-invariant sources emit physical modes only. Moreover, the equations which follow from the requirement that the variation of the Lagrangian with respect to the Goldstone boson field vanishes, are not equations of motion but constraints, expressing the fact that the gauge fields couple to conserved currents. These constraints can also be obtained by taking the derivative of the equations of motion for the gauge fields.

The one-loop contribution to the generating functional can be evaluated with the saddle-point approximation. Because of gauge invariance, the quadratic form in the path integral representation of this contribution has zero eigenvalues. They correspond to fluctuations around the classical fields which are equivalent to infinitesimal gauge transformations. Hence, the expansion of the fluctuations involves eigenvectors of the differential operator with zero and non-zero eigenvalues. In order to evaluate the path integral one has to equip the space of fields with a metric. The volume element associated with this metric yields a nontrivial one-loop contribution to the generating functional. The integration over the zero modes yields the volume factor of the gauge group, which is absorbed by the normalization of the integral. The remaining integral over the non-zero modes is damped by the usual Gaussian factor. It corresponds to the product of the non-zero eigenvalues of the differential operator in the quadratic form. As usual, all one-loop contributions can be expressed in terms of determinants of differential operators. Since the gauge is not fixed, there are no ghost contributions in our approach.

As mentioned above, the classical Goldstone boson field represents the $S U(2)_{L}$ gauge degrees of freedom. Thus, at the classical level only physical modes propagate. At the quantum level, however, the situation is different. Quantum fluctuations around the classical Goldstone boson field imply virtual Goldstone boson modes propagating within loops. Note that these modes are absent in any gauge-dependent approach based on the unitary gauge. They are, however, necessary in order to ensure a decent high energy behavior of the theory.

The one-loop renormalization of the theory was discussed in detail. The Green's functions of currents, scalar densities and field strengths are more singular at short distances than the Green's functions of the fields. The time ordering of these operators gives rise to ambiguities which do not occur for the fields themselves. These ambiguities are reflected by the presence of additional source terms, which enter Green's functions through contact terms. We stress that this is a general feature of any field theory. It is neither particular to our gauge-invariant approach nor to gauge theories in general. Using dimensional regularization and employing heat-kernel techniques we analyzed the short distance properties of the theory. Ultraviolet divergences are related to the poles of the $d$-dimensional determinant which describes the one-loop contributions to the generating functional. With this approach we were able to determine the renormalization prescriptions of the mass parameter and all coupling constants, fields and source terms, independently of any renormalization scheme. Due to the dimension of the source terms involved, the generating functional should be renormalizable at any loop level. Furthermore, the phase factor which was introduced in order to ensure full $S U(2)_{L} \times U(1)_{Y}$ invariance should not spoil the renormalizability of the theory at any loop-level 
either. This is due to the fact that the phase factor only contains the Abelian gauge degree of freedom which does not affect the dynamics of the theory.

Finally, we related the bare parameters of the theory to physical quantities in the on-shell scheme, i.e., we chose the masses of the Higgs, the $W$ - and the $Z$-boson as well as the electric charge as physical input parameters. The masses were defined as pole positions of the two-point functions

$$
\left\langle 0\left|T\left(\Phi^{\dagger} \Phi\right)(x)\left(\Phi^{\dagger} \Phi\right)(y)\right| 0\right\rangle, \quad\left\langle 0\left|T\left(\varphi^{a c} V_{\mu}^{c}\right)(x)\left(\varphi^{b d} V_{\nu}^{d}\right)(y)\right| 0\right\rangle .
$$

The results for the pole masses can be found in Eqs. (6.19), (6.21), and (6.23). The electric charge was defined as the residue at the photon pole of the two-point function

$$
\left\langle 0\left|T B_{\mu \nu}(x) B_{\rho \sigma}(y)\right| 0\right\rangle .
$$

The calculation showed that the result in Eq. (6.25) for the electromagnetic coupling constant defined in this way agrees with the well known result for the electric charge in the Thompson limit. This result is quite interesting since there are no Ward identities in the usual sense between our gauge-invariant Green's functions. Note, that the usual Ward identities relate gauge-dependent Green's functions. Hence, in our approach there is ad hoc no identity relating the residue of the two-point function in Eq. (7.2) at the photon pole to a three-point vertex. We did not evaluate any three-point function in order to check whether it leads to the same result for the coupling constant.

At any rate, the absence of Ward identities does not imply any lack of knowledge. All information that Ward identities encode in any gauge-dependent framework is manifest in our gauge-invariant approach. As an example this was explicitly discussed for the relation between the renormalization factors of the coupling constants $g$ and $g^{\prime}$ and those of the gauge fields corresponding to the symmetry groups $S U(2)_{L}$ and $U(1)_{Y}$, respectively.

We have not included fermions in the present analysis of the standard model. However, the treatment of spin- $1 / 2$ particles in our approach is straightforward. One may choose, for instance, the gauge-invariant fields $\Phi^{\dagger} q_{L}^{k}, \widetilde{\Phi}^{\dagger} q_{L}^{k}, \Phi^{\dagger} l_{L}^{k}$, and $\widetilde{\Phi}^{\dagger} l_{L}^{k}$, cf. Eq. (2.41). The corresponding sources which emit fermionic one-particle states have already been specified in Ref. [17]. As pointed out in Refs. [25,26] the complete screening of the $S U(2)_{L}$ charge of the composite fields $V_{\mu}^{a}, \Phi^{\dagger} \Phi$ and of the fermionic fields written above can also be interpreted as the manifestation of confinement in the electroweak theory, similarly to the mechanism in QCD. As discussed in Ref. 26] the physically observed particles then correspond to "mesonic" and "baryonic" bound states of the usual fields that appear in the electroweak Lagrangian, see also Sec. II. Our approach, extending the gauge-invariant treatment to the full group $S U(2)_{L} \times U(1)_{Y}$, can thus be viewed at as a well-defined framework for carrying out calculations which involve only those external fields which correspond to the physically observed particles $t$.

We note that the application of our approach to other non-Abelian gauge theories like QCD is also possible. However, the structure of the relevant source terms will be different from those used in this article. The definition of, e.g., $S U(2)_{L} \times U(1)_{Y}$ invariant sources exciting fermionic one-particle states as given in Ref. [17] is only possible in the spontaneously broken phase. Hence, an analogous definition does not exist in QCD. Physically, however, it is not necessary either. Since QCD is confining, asymptotic states do not carry any $S U(3)_{c}$ charge. From a physical point of view it should thus be enough to consider Green's functions of $S U(3)_{c}$ invariant operators like, for instance, $\bar{\Psi} \Psi$ or $\operatorname{tr}\left(G_{\mu \nu} G_{\mu \nu}\right)$. Note, however, that the selection of suitable gauge-invariant Green's functions depends on the physical problem one wants to investigate. There is no definite choice which applies to all cases. We believe, however, that a suitable choice should always be possible, since physical quantities are gauge-invariant. Any generating functional for QCD that involves gauge-invariant source terms only can then be evaluated in the perturbative regime with the same technique as described in this article. For attempts to dress $S U(3)_{c}$ charged quarks and gluons with a non-Abelian generalization of our phase factor see Refs. [32, 33].

A first application of our gauge-invariant method can be found in Ref. 19] where we analyzed the electroweak chiral Lagrangian [20], which describes the low-energy structure of a strongly interacting electroweak symmetry breaking sector. In particular, we determined the number of independent parameters in the effective Lagrangian. Furthermore, we evaluated the effective Lagrangian for the standard model with a heavy Higgs boson by matching gauge-invariant Green's functions in the full and the effective theory.

\footnotetext{
${ }^{4}$ Of course, if we switch on the QCD interactions, the quarks will be confined in hadrons.
} 


\section{ACKNOWLEDGMENTS}

We are grateful to F. Jegerlehner and V. Ravindran for enlightening discussions, a careful reading of the manuscript and suggestions for improvements. We are furthermore indebted to J. Gasser, M. Knecht, H. Leutwyler, E. de Rafael, J. Stern, O. Veretin, and A. Vicini for useful discussions. A.N. is grateful to the members of the Yale Physics department for their kind hospitality during the early stages of this project. He also acknowledges financial support by Schweizerischer Nationalfonds during that period.

\section{APPENDIX A: DIFFERENTIAL OPERATORS}

The explicit results for the differential operators which appear in Sec. IV are given below. In the following, upper case Latin indices $A, B, \ldots$ run from 1 to 4 , lower case Latin indices $a, b, \ldots$ run from 1 to 3 , and Greek indices $\alpha, \beta, \ldots$ label the components 1,2 .

The components of the differential operator $\underline{\widetilde{D}}+\underline{P P^{T}}+\delta_{P}$ in Eq. (4.29) are given by

$$
\begin{aligned}
d= & -\square+2 m^{2}+3 m^{2}\left(R^{2}-1\right)+\frac{1}{4} \underline{\mathcal{Y}}_{\mu}^{a} \underline{\mathcal{Y}}_{\mu}^{a}-\widehat{h} \\
\delta^{b}= & -\underline{\mathcal{Y}}_{\rho}^{a} \widehat{\mathcal{D}}_{\rho}^{a b}-\frac{1}{2}\left(\underline{\mathcal{D}}_{\rho} \underline{\mathcal{Y}}_{\rho}\right)^{b} \\
\delta^{T^{a}}= & \underline{\mathcal{Y}}_{\rho}^{a} \partial_{\rho}+\frac{1}{2}\left(\widehat{\mathcal{D}}_{\rho} \underline{\mathcal{Y}}_{\rho}\right)^{a} \\
D^{a b}= & -\left(\widehat{\mathcal{D}}_{\rho} \widehat{\underline{\mathcal{D}}}_{\rho}\right)^{a b}+\delta^{a b}\left(m^{2}\left(R^{2}-1\right)-\widehat{h}\right)+M_{W}^{2} R^{2} \delta^{a b}+\frac{1}{4} \underline{\mathcal{Y}}_{\rho}^{a} \underline{\mathcal{Y}}_{\rho}^{b}, \\
\delta_{\nu}^{B}= & M_{W} R \widetilde{\mathcal{Y}}_{\mu}^{A} \widetilde{\mathrm{PT}}_{\mu \nu}^{A B} \\
\delta_{\mu}^{T, A}= & M_{W} \widetilde{\mathrm{PT}}_{\mu \nu}^{A B} R \widetilde{\mathcal{Y}}_{\nu}^{B} \\
\Delta_{\nu}^{a B}= & f^{a B c} M_{W} R \underline{\mathcal{Y}}_{\nu}^{c}+2 M_{W}\left(\partial_{\nu} R\right) \delta^{a B}-s M_{Z} \delta^{4 B}\left(2 \delta^{a 3}\left(\partial_{\mu} R\right)+R T^{a c} \underline{\mathcal{W}}_{\mu}^{c}\right) \mathrm{PT}_{\mu \nu}, \\
\Delta_{\mu}^{T, A b}= & -f^{A b c} M_{W} R \underline{\mathcal{Y}}_{\mu}^{c}+2 M_{W}\left(\partial_{\mu} R\right) \delta^{A b}+s M_{Z} \delta^{A 4} \mathrm{PT}_{\mu \nu}\left(R \underline{\mathcal{W}}_{\nu}^{c} T^{c b}-2\left(\partial_{\nu} R\right) \delta^{3 b}\right), \\
D_{\mu \nu}^{A B}= & -\delta_{\mu \nu}\left(\widetilde{\mathcal{D}}_{\rho} \widetilde{\mathcal{D}}_{\rho}\right)^{A B}+2 f^{A B}{ }^{A B} \underline{\mathcal{W}}_{\mu \nu}^{c}+\left(\widetilde{M}^{2}\right)^{A B} \mathrm{PT}_{\mu \nu}+M_{W}^{2} \delta^{A B} \mathrm{PL}_{\mu \nu} \\
& +\widetilde{\mathrm{PT}}_{\mu \alpha}^{A C}\left(\widetilde{M}^{2}\right)^{C D}\left(R^{2}-1\right) \widetilde{\mathrm{PT}}_{\alpha \nu}^{D B}+\delta^{A 4} \delta^{B 4} \mathrm{PT}_{\mu \rho} \widehat{J}_{\rho \sigma} \mathrm{PT}_{\sigma \nu},
\end{aligned}
$$

where we introduced the quantities

$$
\begin{aligned}
& \underline{\mathcal{D}}_{\mu}^{a b}=\partial_{\mu} \delta^{a b}-\varepsilon^{a b c}\left(\underline{\mathcal{W}}_{\mu}^{c}-\delta^{3 c} B_{\mu}^{L}\right), \\
& \widehat{\mathcal{D}}_{\mu}^{a b}=\underline{\mathcal{D}}_{\mu}^{a b}+\frac{1}{2} \varepsilon^{a b c} \underline{\mathcal{Y}}_{\mu}^{c}, \\
& \underline{\widetilde{\mathcal{D}}}_{\mu}^{A B}=\partial_{\mu} \delta^{A B}-f^{A B c}\left(\underline{\mathcal{W}}_{\mu}^{c}-\delta^{3 c} B_{\mu}^{L}\right) \text {, } \\
& \tilde{\mathcal{Y}}_{\mu}^{A}=\left(\begin{array}{c}
\mathcal{Y}_{\mu}^{a} \\
-\frac{s}{c} \mathcal{Y}_{\mu}^{3}
\end{array}\right) \\
& \underline{\underline{\mathcal{W}}}_{\mu \nu}^{\alpha}=\partial_{\mu} \underline{\mathcal{W}}_{\nu}^{\alpha}-\partial_{\nu} \underline{\mathcal{W}}_{\mu}^{\alpha}-\left(\mathcal{W}_{\mu}^{3}-B_{\mu}^{L}\right) T_{e}^{\alpha \beta} \underline{\mathcal{W}}_{\nu}^{\beta}+\left(\mathcal{W}_{\nu}^{3}-B_{\nu}^{L}\right) T_{e}^{\alpha \beta} \underline{\mathcal{W}}_{\mu}^{\beta} \text {, } \\
& \underline{\underline{\mathcal{W}}}_{\mu \nu}^{3}=\partial_{\mu} \mathcal{W}_{\nu}^{3}-\partial_{\nu} \mathcal{W}_{\mu}^{3}+\underline{\mathcal{W}}_{\mu}^{\alpha} T_{e}^{\alpha \beta} \underline{\mathcal{W}}_{\nu}^{\beta} \text {, } \\
& T_{e}^{\alpha \beta}=\left(\begin{array}{cc}
0 & 1 \\
-1 & 0
\end{array}\right), \\
& \widetilde{\mathrm{PT}}_{\mu \nu}=\operatorname{diag}\left(\delta_{\mu \nu}, \delta_{\mu \nu}, \delta_{\mu \nu}, \mathrm{PT}_{\mu \nu}\right) \text {, } \\
& \widetilde{M}^{2}=\left(\begin{array}{rrrr}
M_{W}^{2} & 0 & 0 & 0 \\
0 & M_{W}^{2} & 0 & 0 \\
0 & 0 & c^{2} M_{Z}^{2} & -c s M_{Z}^{2} \\
0 & 0 & -c s M_{Z}^{2} & s^{2} M_{Z}^{2}
\end{array}\right), \\
& \widehat{J}_{\mu \nu}=g^{\prime 2} v_{d j}\left(\delta_{\mu \nu} J_{\kappa}^{\alpha} J_{\kappa}^{\alpha}-J_{\mu}^{\alpha} J_{\nu}^{\alpha}\right) \text {. }
\end{aligned}
$$


Note that the combination $\mathcal{W}_{\mu}^{3}-B_{\mu}^{L}$ which appears in the expressions above is $S U(2)_{L} \times U(1)_{Y}$ gauge-invariant, since

$$
\mathcal{W}_{\mu}^{3}-B_{\mu}^{L}=\mathcal{Z}_{\mu}+s^{2} \mathcal{Z}_{\mu}^{T}-\mathcal{A}_{\mu}^{T} .
$$

Using the definition from Eq. (4.21) of the operator $P$ which creates zero modes, we obtain the following expressions for the differential operators $\underline{P P^{T}}$ and $\underline{P^{T} P}$ which appear in Eq. (4.28):

$$
\begin{aligned}
& \underline{P P^{T}}=\left(\begin{array}{ccc}
0 & 0 & 0 \\
0 & M_{W}^{2} R^{2} \delta^{a b} & -M_{W} R \widetilde{\mathcal{D}}_{\nu}^{a B} \\
0 & M_{W} \widetilde{\mathcal{D}}_{\mu}^{A b} R & -\left(\widetilde{\mathcal{D}}_{\mu} \widetilde{\mathcal{D}}_{\nu}\right)^{A B}
\end{array}\right), \\
& \underline{P^{T} P}=\left(\begin{array}{cc}
-\underline{\mathcal{D}}_{\mu}^{a c} \underline{\mathcal{D}}_{\mu}^{c b}+M_{W}^{2} R^{2} \delta^{a b} & 0 \\
0 & -\square
\end{array}\right) .
\end{aligned}
$$

Furthermore, the operator $\delta_{P}$ is defined by

$$
\delta_{P}=\operatorname{diag}\left(0,0, \delta^{A 4} \delta^{4 B} M_{W}^{2} \mathrm{PL}_{\mu \nu}\right) .
$$

The operators $\delta_{\mu}$ and $\Delta_{\mu}$ which appear in Eqs. (4.39)-(4.41) are given by the following expressions:

$$
\begin{aligned}
\delta_{\nu}^{\mathcal{W}^{\alpha}} & =M_{W} R \underline{\mathcal{Y}}_{\nu}^{\alpha}, \\
\delta_{\nu}^{\mathcal{Z}} & =M_{Z} R \mathcal{Y}_{\mu}^{3}\left(c^{2} \delta_{\mu \nu}+s^{2} \mathrm{PT}_{\mu \nu}\right), \\
\delta_{\nu}^{\mathcal{A}} & =s c M_{Z} R \mathcal{Y}_{\mu}^{3} \mathrm{PL}_{\mu \nu}, \\
\delta_{\mu}^{\mathcal{W}, T^{\beta}} & =M_{W} R \underline{\mathcal{Y}}_{\mu}^{\beta}, \\
\delta_{\mu}^{\mathcal{Z}, T} & =M_{Z}\left(c^{2} \delta_{\mu \nu}+s^{2} \mathrm{PT}_{\mu \nu}\right) \mathcal{Y}_{\nu}^{3} R, \\
\delta_{\mu}^{\mathcal{A}, T} & =s c M_{Z} \mathrm{PL}_{\mu \nu} \mathcal{Y}_{\nu}^{3} R \\
\Delta_{\nu}^{\mathcal{W}}{ }^{a \beta} & =\varepsilon^{a \beta c} M_{W} R \underline{\mathcal{Y}}_{\nu}^{c}+2 M_{W}\left(\partial_{\nu} R\right) \delta^{a \beta}, \\
\Delta_{\mu}^{\mathcal{W}, T^{\alpha b}} & =-\varepsilon^{\alpha b c} M_{W} R \underline{\mathcal{Y}}_{\mu}^{c}+2 M_{W}\left(\partial_{\mu} R\right) \delta^{\alpha b}, \\
\Delta_{\nu}^{\mathcal{Z}} & =\varepsilon^{a 3 c} M_{Z} R\left(c^{2} \underline{\mathcal{Y}}_{\nu}^{c}-s^{2} \underline{\mathcal{W}}_{\mu}^{c} \mathrm{PT}_{\mu \nu}\right)+2 \delta^{a 3} M_{Z}\left(\partial_{\mu} R\right)\left(c^{2} \delta_{\mu \nu}+s^{2} \mathrm{PT}_{\mu \nu}\right), \\
\Delta_{\nu}^{\mathcal{A}}{ }^{a} & =\varepsilon^{a 3 c} s c M_{Z} R\left(\underline{\mathcal{Y}}_{\nu}^{c}+\underline{\mathcal{W}}_{\mu}^{c} \mathrm{PT}_{\mu \nu}\right)+2 s c \delta^{a 3} M_{Z}\left(\partial_{\mu} R\right) \mathrm{PL}_{\mu \nu}, \\
\Delta_{\mu}^{\mathcal{Z}, T^{b}} & =-\varepsilon^{3 b c} M_{Z}\left(c^{2} \underline{\mathcal{Y}}_{\mu}^{c}-s^{2} \mathrm{PT}_{\mu \nu} \underline{\mathcal{W}}_{\nu}^{c}\right) R+2 \delta^{3 b} M_{Z}\left(c^{2} \delta_{\mu \nu}+s^{2} \mathrm{PT}_{\mu \nu}\right)\left(\partial_{\nu} R\right), \\
\Delta_{\mu}^{\mathcal{A}, T^{b}} & =-\varepsilon^{3 b c} s c M_{Z}\left(\underline{\mathcal{Y}}_{\mu}^{c}+\mathrm{PT}_{\mu \nu} \underline{\mathcal{W}}_{\nu}^{c}\right) R+2 s c \delta^{3 b} M_{Z} \mathrm{PL}_{\mu \nu}\left(\partial_{\nu} R\right) .
\end{aligned}
$$

The first terms on the right-hand side of Eqs. (4.39)-(4.41) read as follows:

$$
\begin{aligned}
& D_{\mu \nu}^{\mathcal{\mathcal { W } ^ { \alpha \beta }}}=D_{\mu \nu}^{W, 0^{\alpha \beta}}+T_{e}^{\alpha \beta}\left(\left(\partial_{\sigma}\left(\mathcal{W}_{\sigma}^{3}-B_{\sigma}^{L}\right)\right)+2\left(\mathcal{W}_{\sigma}^{3}-B_{\sigma}^{L}\right) \partial_{\sigma}\right) \delta_{\mu \nu}-\left(T_{e}^{\alpha \gamma} \underline{\mathcal{W}}_{\sigma}^{\gamma}\right)\left(\underline{\mathcal{W}}_{\sigma}^{\delta} T_{e}^{\delta \beta}\right) \delta_{\mu \nu} \\
& +\left(\mathcal{W}_{\sigma}^{3}-B_{\sigma}^{L}\right)\left(\mathcal{W}_{\sigma}^{3}-B_{\sigma}^{L}\right) \delta_{\mu \nu} \delta^{\alpha \beta}+2 T_{e}^{\alpha \beta} \underline{\underline{\mathcal{W}}}_{\mu \nu}^{3}+M_{W}^{2}\left(R^{2}-1\right) \delta_{\mu \nu} \delta^{\alpha \beta}, \\
& D_{\mu \nu}^{\mathcal{Z}}=D_{\mu \nu}^{\mathcal{Z}, 0}+c^{2} \underline{\mathcal{W}}_{\sigma}^{\alpha} \underline{\mathcal{W}}_{\sigma}^{\alpha} \delta_{\mu \nu}+s^{2} \mathrm{PT}_{\mu \rho} \widehat{J}_{\rho \sigma} \mathrm{PT}_{\sigma \nu} \\
& +M_{Z}^{2}\left(c^{2} \delta_{\mu \rho}+s^{2} \mathrm{PT}_{\mu \rho}\right)\left(R^{2}-1\right)\left(c^{2} \delta_{\rho \nu}+s^{2} \mathrm{PT}_{\rho \nu}\right), \\
& D_{\mu \nu}^{\mathcal{A}}=D_{\mu \nu}^{\mathcal{A}, 0}+s^{2} \underline{\mathcal{W}}_{\sigma}^{\alpha} \underline{\mathcal{W}}_{\sigma}^{\alpha} \delta_{\mu \nu}+s^{2} M_{W}^{2} \mathrm{PL}_{\mu \rho}\left(R^{2}-1\right) \mathrm{PL}_{\rho \nu}+c^{2} \mathrm{PT}_{\mu \rho} \widehat{J}_{\rho \sigma} \mathrm{PT}_{\sigma \nu} \\
& \xi_{\mu \nu}^{\mathcal{Z}}{ }^{\alpha}=-c T_{e}^{\alpha \beta}\left(\left(\partial_{\sigma} \underline{\mathcal{W}}_{\sigma}^{\beta}\right)+2 \underline{\mathcal{W}}_{\sigma}^{\beta} \partial_{\sigma}\right) \delta_{\mu \nu}-c\left(\mathcal{W}_{\sigma}^{3}-B_{\sigma}^{L}\right) \underline{\mathcal{W}}_{\sigma}^{\alpha} \delta_{\mu \nu}-2 c T_{e}^{\alpha \beta} \underline{\underline{\mathcal{W}}}_{\mu \nu}^{\beta}, \\
& \xi_{\mu \nu}^{\mathcal{Z}, T^{\beta}}=-c\left(\left(\partial_{\sigma} \underline{\mathcal{W}}_{\sigma}^{\alpha}\right)+2 \underline{\mathcal{W}}_{\sigma}^{\alpha} \partial_{\sigma}\right) T_{e}^{\alpha \beta} \delta_{\mu \nu}-c\left(\mathcal{W}_{\sigma}^{3}-B_{\sigma}^{L}\right) \underline{\mathcal{W}}_{\sigma}^{\beta} \delta_{\mu \nu}-2 c \underline{\underline{\mathcal{W}}}_{\mu \nu}^{\alpha} T_{e}^{\alpha \beta}, \\
& \xi_{\mu \nu}^{\mathcal{A}}{ }^{\alpha}=-s T_{e}^{\alpha \beta}\left(\left(\partial_{\sigma} \underline{\mathcal{W}}_{\sigma}^{\beta}\right)+2 \underline{\mathcal{W}}_{\sigma}^{\beta} \partial_{\sigma}\right) \delta_{\mu \nu}-s\left(\mathcal{W}_{\sigma}^{3}-B_{\sigma}^{L}\right) \underline{\mathcal{W}}_{\sigma}^{\alpha} \delta_{\mu \nu}-2 s T_{e}^{\alpha \beta} \underline{\underline{\mathcal{W}}}_{\mu \nu}^{\beta}, \\
& \xi_{\mu \nu}^{\mathcal{A}, T^{\beta}}=-s\left(\left(\partial_{\sigma} \underline{\mathcal{W}}_{\sigma}^{\alpha}\right)+2 \underline{\mathcal{W}}_{\sigma}^{\alpha} \partial_{\sigma}\right) T_{e}^{\alpha \beta} \delta_{\mu \nu}-s\left(\mathcal{W}_{\sigma}^{3}-B_{\sigma}^{L}\right) \underline{\mathcal{W}}_{\sigma}^{\beta} \delta_{\mu \nu}-2 s \underline{\underline{\mathcal{W}}}_{\mu \nu}^{\alpha} T_{e}^{\alpha \beta}, \\
& \eta_{\mu \nu}^{\mathcal{A}}=s c \underline{\mathcal{W}}_{\sigma}^{\alpha} \underline{\mathcal{W}}_{\sigma}^{\alpha} \delta_{\mu \nu}+s c M_{Z}^{2}\left(c^{2} \delta_{\mu \rho}+s^{2} \mathrm{PT}_{\mu \rho}\right)\left(R^{2}-1\right) \mathrm{PL}_{\rho \nu}-s c \mathrm{PT}_{\mu \rho} \widehat{J}_{\rho \sigma} \mathrm{PT}_{\sigma \nu}, \\
& \eta_{\mu \nu}^{\mathcal{A}, T}=s c \underline{\mathcal{W}}_{\sigma}^{\alpha} \underline{\mathcal{W}}_{\sigma}^{\alpha} \delta_{\mu \nu}+s c M_{Z}^{2} \mathrm{PL}_{\mu \rho}\left(R^{2}-1\right)\left(c^{2} \delta_{\rho \nu}+s^{2} \mathrm{PT}_{\rho \nu}\right)-s c \mathrm{PT}_{\mu \rho} \widehat{J}_{\rho \sigma} \mathrm{PT}_{\sigma \nu} .
\end{aligned}
$$


Free propagators are the inverse of the following operators:

$$
\begin{aligned}
d^{0} & \equiv d_{m}=-\square+2 m^{2}, \\
\Theta^{0^{a b}} & \equiv D^{0^{a b}}=\left(-\square+M_{W}^{2}\right) \delta^{a b}, \\
\mathcal{D}_{\mu \nu}^{\mathcal{W}, 0^{\alpha \beta}} & \equiv D_{\mu \nu}^{\mathcal{W}, 0}=\left(-\square+M_{W}^{2}\right) \delta_{\mu \nu} \delta^{\alpha \beta}, \\
\mathcal{D}_{\mu \nu}^{\mathcal{Z}, 0} & \equiv D_{\mu \nu}^{\mathcal{Z}, 0}=\left(-\square+M_{Z}^{2}\right) \mathrm{PT}_{\mu \nu}+\left(-\square+M_{W}^{2}\right) \mathrm{PL}_{\mu \nu}, \\
\mathcal{D}_{\mu \nu}^{\mathcal{A}, 0} & \equiv D_{\mu \nu}^{\mathcal{A}, 0}=-\square \mathrm{PT}_{\mu \nu}+\left(-\square+M_{W}^{2}\right) \mathrm{PL}_{\mu \nu},
\end{aligned}
$$

which are obtained in the limit where all sources are switched off. We observe that all transversal components propagate with the proper mass, while all longitudinal components propagate with the $W$-boson mass.

Since we perform a saddle-point approximation in the path integral, the fields which appear in the list of differential operators in Eqs. (A1)-(A44) obey the equations of motion (3.2)-(3.7). We have used this fact to simplify the expressions of those operators in Eqs. (A1) A9 which correspond to the fluctuations $\eta^{a}$ of the Goldstone bosons.

Furthermore, it is important to ensure that the full differential operator $\underline{\widetilde{D}}+\underline{P P^{T}}+\delta_{P}$ is Hermitian, i.e. satisfies the relation $\left(y,\left[\underline{\widetilde{D}}+\underline{P P^{T}}+\delta_{P}\right] y^{\prime}\right)=\left(y^{\prime},\left[\underline{\widetilde{D}}+\underline{P P^{T}}+\delta_{P}\right] y\right)$ for arbitrary fluctuation vectors $y, y^{\prime}$.

\section{APPENDIX B: RENORMALIZATION PRESCRIPTIONS}

For the calculation of the counterterms and the renormalization prescriptions it is convenient to switch to a real $O(4)$-representation for the Higgs field:

$$
\phi=\left(\begin{array}{l}
\phi^{1} \\
\phi^{2} \\
\phi^{3} \\
\phi^{4}
\end{array}\right) .
$$

The covariant derivative for the Higgs field is given by

$$
\begin{aligned}
\nabla_{\mu}^{N M} \phi^{M} & =\left(\partial_{\mu} \delta^{N M}+F_{\mu}^{N M}\right) \phi^{M}, \quad N, M=1,2,3,4, \\
F_{\mu} & =W_{\mu}^{a} T_{L}^{a}+B_{\mu} T_{R}^{3}, \quad a=1,2,3,
\end{aligned}
$$

where the matrices $T_{L, R}$ are defined through

$$
\begin{aligned}
& T_{R}^{a}=\frac{1}{2}\left(\widehat{T}^{a}+\widetilde{T}^{a}\right), \quad T_{L}^{a}=\frac{1}{2}\left(\widehat{T}^{a}-\widetilde{T}^{a}\right), \\
&\left(\widehat{T}_{c}\right)^{N M} \doteq-\varepsilon^{N M c}, \quad\left(\widetilde{T}_{c}\right)^{N M} \doteq \delta_{4}^{N} \delta_{c}^{M}-\delta_{4}^{M} \delta_{c}^{N} .
\end{aligned}
$$

It can easily be shown that the matrices $T_{R}^{a}$ and $T_{L}^{a}$ separately satisfy the Pauli algebra. The Lagrangian in Eq. (2.25) can then be rewritten in terms of the fields in the real representation. The main changes compared to the notation with a complex doublet $\Phi$ are obtained by the following replacements:

$$
\begin{aligned}
\frac{1}{2} D_{\mu} \Phi^{\dagger} D_{\mu} \Phi & \rightarrow \frac{1}{2} \nabla_{\mu} \phi^{T} \nabla_{\mu} \phi \quad, \quad \Phi^{\dagger} \Phi \rightarrow \phi^{T} \phi, \\
J_{\mu}^{a} \varphi^{a b} V_{\mu}^{b} & \rightarrow 4 J_{\mu}^{a} \varphi^{a b} \mathcal{J}_{R \mu}^{b},
\end{aligned}
$$

with

$$
\mathcal{J}_{R \mu}^{a}=\phi^{T} T_{R}^{a} \nabla_{\mu} \phi
$$

The evaluation of the path integral representation of the generating functional in Eq. (4.1) is again performed by a saddle-point approximation around the classical action. The quantum fluctuations are introduced as simple shifts in the fields

$$
\begin{aligned}
\phi^{N} & \rightarrow \phi^{N}+f^{N}, \\
F_{\mu} & \rightarrow F_{\mu}+g w_{\mu}^{a} T_{R}^{a}+g^{\prime} b_{\mu} T_{L}^{3} .
\end{aligned}
$$


It will be useful to treat the fluctuations $w_{\mu}^{a}, b_{\mu}$ and the matrices $T_{L}^{a}, T_{R}^{3}$ in a unified way by introducing the quantities:

$$
q_{\mu}^{A} \doteq\left(\begin{array}{c}
w_{\mu}^{a} \\
b_{\mu}
\end{array}\right), \quad t^{A} \doteq\left(\begin{array}{c}
T_{L}^{a} \\
T_{R}^{3}
\end{array}\right), \quad A=1,2,3,4
$$

The terms quadratic in the fluctuations determine the differential operator $\widetilde{D}+\bar{P} \bar{P}^{T}$, after the proper treatment of the zero modes. From the path integral measure one gets the operator $\bar{P}^{T} \bar{P}$. The final result for these operators can be written in the following way, which is suitable for using the heat-kernel method and the short distance expansion outlined in Sec. V,

$$
\widetilde{D}+\bar{P} \bar{P}^{T}=D_{\mathrm{loc}}+\delta D,
$$

where the local part $D_{\text {loc }}$ of the differential operator is given by

$$
\begin{aligned}
D_{\mathrm{loc}}= & -\bar{D}_{\rho} \bar{D}_{\rho}+\bar{\sigma}, \\
\bar{D}_{\rho}= & \left(\begin{array}{cc}
\bar{d}_{\rho} & 0 \\
0 & \delta_{\mu \nu} \overline{\mathcal{D}}_{\rho}
\end{array}\right), \\
\bar{d}_{\rho}^{N M}= & \delta^{N M} \partial_{\rho}+F_{\rho}^{N M}-4 j_{\rho}^{N M}, \\
\overline{\mathcal{D}}_{\rho}^{A B}= & \delta^{A B} \partial_{\rho}-f^{A B c} W_{\rho}^{c}, \\
\bar{\sigma}= & \left(\begin{array}{cc}
\bar{\sigma}_{f f} & \bar{\sigma}_{f q} \\
\bar{\sigma}_{q f} & \bar{\sigma}_{q q}
\end{array}\right), \\
\bar{\sigma}_{f f}^{N M}= & \left(-m^{2}+\lambda \phi^{T} \phi-h-v_{j j} J_{\mu}^{\alpha} J_{\mu}^{\alpha}-c_{j j} J_{\mu}^{Z} J_{\mu}^{Z}\right) \delta^{N M} \\
& +2 \lambda \phi^{N} \phi^{M}-\left(t^{A} \phi \otimes \phi^{T} t^{A}\right)^{N M}+16\left(j_{\rho} j_{\rho}\right)^{N M}, \\
\bar{\sigma}_{f q, \nu}^{N B}= & -2\left(t^{B} \nabla_{\nu} \phi\right)^{N}+4\left\{j_{\nu}, t^{B}\right\}^{N M} \phi^{M}, \\
\bar{\sigma}_{q f, \mu}^{A M}= & 2\left(\nabla_{\mu} \phi^{T} t^{A}\right)^{M}+4 \phi^{N}\left\{j_{\mu}, t^{A}\right\}^{N M}, \\
\bar{\sigma}_{q q, \mu \nu}^{A B}= & -\delta_{\mu \nu} \phi^{T} t^{A} t^{B} \phi-2 W_{\mu \nu}^{A d j} A B+\delta^{A 4} \delta^{B 4} \widehat{J}_{\mu \nu} .
\end{aligned}
$$

Here we introduced the quantities

$$
\begin{aligned}
j_{\mu} & =j_{\mu}^{a} T_{R}^{a}, \\
W_{\mu \nu}^{A d j}{ }^{A B} & =\left[\overline{\mathcal{D}}_{\mu}, \overline{\mathcal{D}}_{\nu}\right]^{A B}=-f^{A B c} W_{\mu \nu}^{c} .
\end{aligned}
$$

The quantities $f^{A B c}$ and $\widehat{J}_{\mu \nu}$ have been defined in Eqs. (4.22) and (A19), respectively.

The nonlocal part $\delta D$ of the differential operator which contains the contributions from the phase factor reads

$$
\begin{aligned}
\delta D= & \left(\begin{array}{cc}
0 & \delta D_{f q} \\
\delta D_{q f} & \delta D_{q q}
\end{array}\right), \\
\delta D_{f q, \nu}^{N B}= & -4 g^{\prime} \delta^{B 4}\left(2 j_{\rho}^{\alpha} T_{e}^{\alpha \beta} T_{R}^{\beta} \nabla_{\rho} \phi+\left(d_{\rho} j_{\rho}\right)^{\alpha} T_{e}^{\alpha \beta} T_{R}^{\beta} \phi\right)^{N}\left(\partial_{\nu} \frac{1}{\square}\right)-4 g^{\prime} \delta^{B 4}\left(j_{\rho}^{\alpha} T_{e}^{\alpha \beta} T_{R}^{\beta} \phi\right)^{N}\left(\partial_{\rho} \partial_{\nu} \frac{1}{\square}\right), \\
\delta D_{q f, \mu}^{A M}= & 4 g^{\prime} \delta^{A 4}\left(\partial_{\mu} \frac{1}{\square}\right)\left(\phi^{T} j_{\rho}^{\alpha} T_{e}^{\alpha \beta} T_{R}^{\beta} \nabla_{\rho}-\left(\nabla_{\mu} \phi\right)^{T} j_{\rho}^{\alpha} T_{e}^{\alpha \beta} T_{R}^{\beta}\right)^{M}, \\
\delta D_{q q, \mu \nu}^{A B}= & 8 g^{\prime} \delta^{A 4}\left(\partial_{\mu} \frac{1}{\square}\right)\left(\phi^{T} j_{\nu}^{\alpha} T_{e}^{\alpha \beta} T_{R}^{\beta} t^{B} \phi\right)+4 g^{\prime 2} \delta^{A 4} \delta^{B 4}\left(\partial_{\mu} \frac{1}{\square}\right)\left(\phi^{T} j_{\rho}^{\alpha} T_{R}^{\alpha} \nabla_{\rho} \phi\right)\left(\partial_{\nu} \frac{1}{\square}\right) \\
& -\delta^{A 4} \delta^{B 4}\left(\partial_{\mu} \partial_{\sigma} \frac{1}{\square}\right) \widehat{J}_{\sigma \nu}-\delta^{A 4} \delta^{B 4} \widehat{J}_{\mu \sigma}\left(\partial_{\sigma} \partial_{\nu} \frac{1}{\square}\right)+\delta^{A 4} \delta^{B 4}\left(\partial_{\mu} \partial_{\sigma} \frac{1}{\square}\right) \widehat{J}_{\sigma \kappa}\left(\partial_{\kappa} \partial_{\nu} \frac{1}{\square}\right) .
\end{aligned}
$$

The contributions to the counterterms from the path integral measure can be calculated from the expression

$$
\left(\bar{P}^{T} \bar{P}\right)^{A B}=-\left(\overline{\mathcal{D}}_{\rho} \overline{\mathcal{D}}_{\rho}\right)^{A B}-\phi^{T} t^{A} t^{B} \phi .
$$

The determination of the counterterms then proceeds along the lines sketched in Sec. $\mathrm{V}$. From the counterterms we can read off the renormalization prescriptions for the fields, the mass parameter $m^{2}$, the coupling constants and 
the sources. The relations between bare and renormalized fields, masses and coupling constants which are needed in Sec. $\mathrm{VI}$ are given by

$$
\begin{aligned}
W_{\mu}^{a} & =W_{\mu}^{a, r} \\
B_{\mu} & =B_{\mu}^{r} \\
\phi & =Z_{\phi}^{1 / 2} \phi_{r} \\
Z_{\phi} & =1-\left(6 g_{r}^{2}+2 g_{r}^{\prime 2}\right)\left(\Lambda_{\varepsilon}\left(2 m_{r}^{2}\right)+\delta z\right), \\
m^{2} & =m_{r}^{2}\left[1-\frac{1}{2}\left(24 \lambda_{r}+3 g_{r}^{2}+g_{r}^{\prime 2}\right)\left(\Lambda_{\varepsilon}\left(2 m_{r}^{2}\right)+\delta m^{2}\right)-\left(Z_{\phi}-1\right)\right], \\
\lambda & =\lambda_{r}\left[1-\left(24 \lambda_{r}+3 g_{r}^{2}+g_{r}^{\prime 2}+\frac{3}{8} \frac{\left(g_{r}^{2}+g_{r}^{\prime 2}\right)^{2}+2 g_{r}^{4}}{\lambda_{r}}\right)\left(\Lambda_{\varepsilon}\left(2 m_{r}^{2}\right)+\delta \lambda\right)-2\left(Z_{\phi}-1\right)\right], \\
g^{2} & =g_{r}^{2}\left[1+\frac{43}{3} g_{r}^{2}\left(\Lambda_{\varepsilon}\left(2 m_{r}^{2}\right)+\delta g^{2}\right)\right], \\
g^{\prime 2} & =g_{r}^{\prime 2}\left[1-\frac{1}{3} g_{r}^{\prime 2}\left(\Lambda_{\varepsilon}\left(2 m_{r}^{2}\right)+\delta g^{\prime 2}\right)\right],
\end{aligned}
$$

where we denoted the pole term by

$$
\Lambda_{\varepsilon}\left(2 m_{r}^{2}\right) \doteq \frac{\mu^{d-4}}{16 \pi^{2}}\left(\frac{1}{d-4}-\frac{1}{2}\left(\ln (4 \pi)+\Gamma^{\prime}(1)+1\right)\right)+\frac{1}{32 \pi^{2}} \ln \left(\frac{2 m_{r}^{2}}{\mu^{2}}\right)
$$

The finite renormalization constants $\delta m^{2}, \ldots, \delta g^{\prime 2}$ which appear in the Eqs. (B30)-(B33) are determined by the renormalization conditions given in Sec. VI.

The renormalization of the source terms can be achieved by using the following prescriptions:

$$
\begin{aligned}
h= & c_{h} h_{r} \\
c_{h}= & 1-\frac{1}{2}\left(24 \lambda_{r}+3 g_{r}^{2}+g_{r}^{\prime 2}\right)\left(\Lambda_{\varepsilon}\left(2 m_{r}^{2}\right)+\delta c_{h}\right)-\left(Z_{\phi}-1\right), \\
K_{\mu \nu}= & K_{\mu \nu}^{r} \\
J_{\mu}^{\alpha}= & c_{v} J_{\mu}^{\alpha, r} \\
c_{v}= & 1-\frac{1}{4}\left(24 g_{r}^{2}+2 g_{r}^{\prime 2}\right)\left(\Lambda_{\varepsilon}\left(2 m_{r}^{2}\right)+\delta c_{v}\right)-\left(Z_{\phi}-1\right), \\
J_{\mu}^{Z}= & c_{Z} J_{\mu}^{Z r} \\
c_{Z}= & 1-\left(6 g_{r}^{2}+2 g_{r}^{\prime 2}\right)\left(\Lambda_{\varepsilon}\left(2 m_{r}^{2}\right)+\delta c_{Z}\right)-\left(Z_{\phi}-1\right), \\
v_{j j}= & v_{j j}^{r}+\left(24 g_{r}^{2}+2 g_{r}^{\prime 2}+\frac{9}{8} v_{d j}^{r} g_{r}^{\prime 4}-\frac{1}{2}\left(v_{j j}^{r}+4\right)\left(24 \lambda_{r}+3 g_{r}^{2}+g_{r}^{\prime 2}\right)\right)\left(\Lambda_{\varepsilon}\left(2 m_{r}^{2}\right)+\delta v_{j j}\right) \\
& -v_{j j}^{r}\left(Z_{\phi}-1\right)-2 v_{j j}^{r}\left(c_{v}-1\right) \\
c_{j j}= & c_{j j}^{r}+\left(24 g_{r}^{2}+8 g_{r}^{\prime 2}-\frac{1}{2}\left(c_{j j}^{r}+4\right)\left(24 \lambda_{r}+3 g_{r}^{2}+g_{r}^{\prime 2}\right)\right)\left(\Lambda_{\varepsilon}\left(2 m_{r}^{2}\right)+\delta c_{j j}\right)-c_{j j}^{r}\left(Z_{\phi}-1\right)-2 c_{j j}^{r}\left(c_{Z}-1\right), \\
c_{B j}= & c_{B j}^{r}+\frac{4}{3}\left(\Lambda_{\varepsilon}\left(2 m_{r}^{2}\right)+\delta c_{B j}\right)-c_{B j}^{r}\left(c_{Z}-1\right), \\
c_{B j j}= & c_{B j j}^{r}-\frac{16}{3}\left(\Lambda_{\varepsilon}\left(2 m_{r}^{2}\right)+\delta c_{B j j}\right)-2 c_{B j j}^{r}\left(c_{v}-1\right), \\
v_{d j j}= & v_{d j j}^{r}-\frac{128}{3}\left(\Lambda_{\varepsilon}\left(2 m_{r}^{2}\right)+\delta v_{d j j}\right)-v_{d j j}^{r}\left(c_{Z}-1\right)-2 v_{d j j}^{r}\left(c_{v}-1\right), \\
v_{d j}= & v_{d j}^{r}+\frac{16}{3}\left(\Lambda_{\varepsilon}\left(2 m_{r}^{2}\right)+\delta v_{d j}\right)-2 v_{d j}^{r}\left(c_{v}-1\right), \\
c_{d j j}= & c_{d j j}^{r}-\frac{128}{3}\left(\Lambda_{\varepsilon}\left(2 m_{r}^{2}\right)+\delta c_{d j j}\right)-c_{d j j}^{r}\left(c_{Z}-1\right)-2 c_{d j j}^{r}\left(c_{v}-1\right), \\
c_{d j}= & c_{d j}^{r}+\frac{16}{3}\left(\Lambda_{\varepsilon}\left(2 m_{r}^{2}\right)+\delta c_{d j}\right)-2 c_{d j}^{r}\left(c_{Z}-1\right),
\end{aligned}
$$




$$
\begin{aligned}
v_{J J 2} & =v_{J J 2}^{r}-\left(\frac{37}{48}\left(v_{d j}^{r}\right)^{2} g_{r}^{\prime 4}+2\left(v_{j j}^{r}+4\right)^{2}-\frac{64}{3}\right)\left(\Lambda_{\varepsilon}\left(2 m_{r}^{2}\right)+\delta v_{J J 2}\right)-4 v_{J J 2}^{r}\left(c_{v}-1\right), \\
v_{J J J J} & =v_{J J J}^{r}-\left(\frac{7}{24}\left(v_{d j}^{r}\right)^{2} g_{r}^{\prime 4}+\frac{64}{3}\right)\left(\Lambda_{\varepsilon}\left(2 m_{r}^{2}\right)+\delta v_{J J J J}\right)-4 v_{J J J J}^{r}\left(c_{v}-1\right), \\
c_{J J 2} & =c_{J J 2}^{r}-2\left(c_{j j}^{r}+4\right)^{2}\left(\Lambda_{\varepsilon}\left(2 m_{r}^{2}\right)+\delta c_{J J 2}\right)-4 c_{J J 2}^{r}\left(c_{Z}-1\right), \\
v_{J 2 Z Z} & =v_{J 2 Z Z}^{r}-\left(4\left(v_{j j}^{r}+4\right)\left(c_{j j}^{r}+4\right)-\frac{128}{3}\right)\left(\Lambda_{\varepsilon}\left(2 m_{r}^{2}\right)+\delta v_{J 2 Z Z}\right)-2 v_{J 2 Z Z}^{r}\left(c_{v}-1\right)-2 v_{J 2 Z Z}^{r}\left(c_{Z}-1\right), \\
v_{J J Z Z} & =v_{J J Z Z}^{r}-\frac{128}{3}\left(\Lambda_{\varepsilon}\left(2 m_{r}^{2}\right)+\delta v_{J J Z Z}\right)-2 v_{J J Z Z}^{r}\left(c_{v}-1\right)-2 v_{J J Z Z}^{r}\left(c_{Z}-1\right), \\
c_{h h} & =c_{h h}^{r}-2\left(\Lambda_{\varepsilon}\left(2 m_{r}^{2}\right)+\delta c_{h h}\right)-2 c_{h h}^{r}\left(c_{h}-1\right), \\
c_{m h} & =c_{m h}^{r}-4\left(\Lambda_{\varepsilon}\left(2 m_{r}^{2}\right)+\delta c_{m h}\right)-c_{m h}^{r}\left(c_{h}-1\right)-c_{m h}^{r}\left(\frac{m^{2}-m_{r}^{2}}{m_{r}^{2}}\right) \\
c_{h J J} & =c_{h J J}^{r}-4\left(v_{j j}^{r}+4\right)\left(\Lambda_{\varepsilon}\left(2 m_{r}^{2}\right)+\delta c_{h J J}\right)-c_{h J J}^{r}\left(c_{h}-1\right)-2 c_{h J J}^{r}\left(c_{v}-1\right), \\
c_{h Z Z} & =c_{h Z Z}^{r}-4\left(c_{j j}^{r}+4\right)\left(\Lambda_{\varepsilon}\left(2 m_{r}^{2}\right)+\delta c_{h Z Z}\right)-c_{h Z Z}^{r}\left(c_{h}-1\right)-2 c_{h Z Z}^{r}\left(c_{Z}-1\right), \\
c_{m J J} & =c_{m J J}^{r}-4\left(v_{j j}^{r}+4\right)\left(\Lambda_{\varepsilon}\left(2 m_{r}^{2}\right)+\delta c_{m J J}\right)-2 c_{m J J}^{r}\left(c_{v}-1\right)-c_{m J J}^{r}\left(\frac{m^{2}-m_{r}^{2}}{m_{r}^{2}}\right) \\
c_{m Z Z} & =c_{m Z Z}^{r}-4\left(c_{j j}^{r}+4\right)\left(\Lambda_{\varepsilon}\left(2 m_{r}^{2}\right)+\delta c_{m Z Z}\right)-2 c_{m Z Z}^{r}\left(c_{Z}-1\right)-c_{m Z Z}^{r}\left(\frac{m^{2}-m_{r}^{2}}{m_{r}^{2}}\right) .
\end{aligned}
$$

In order to renormalize on-shell quantities, like masses, coupling constants and $S$-matrix elements it will not be necessary to determine all the finite renormalization constants $\delta c$ and $\delta v$ which appear in Eqs. (B35)-(B60).

\section{APPENDIX C: SELF-ENERGIES}

The explicit results for the self-energies which appear in the generating functional in Eq. (6.9) are given by the following expressions:

$$
\begin{aligned}
\Sigma_{\Phi^{\dagger} \Phi}\left(p^{2}\right)= & \frac{e^{2}}{s^{2} c^{2}}\left\{\left(\frac{3}{4} \frac{2 m^{2}}{M_{Z}^{2}}\right) A_{0}\left(2 m^{2}\right)+\left(\frac{1}{4} \frac{p^{2}}{M_{Z}^{2}}+\frac{3}{4} \frac{2 m^{2}}{M_{Z}^{2}}+\frac{13}{4} \frac{M_{W}^{2}}{M_{Z}^{2}}-\frac{1}{4}\right) A_{0}\left(M_{W}^{2}\right)\right. \\
& +\left(-\frac{1}{4} \frac{p^{2}}{M_{Z}^{2}}+\frac{7}{4}-\frac{1}{4} \frac{M_{W}^{2}}{M_{Z}^{2}}\right) A_{0}\left(M_{Z}^{2}\right) \\
& +\left(-\frac{1}{4} s^{2} c^{2} M_{Z}^{2}\right) B_{0}\left(0, M_{W}^{2} ; 0\right)+\left(\frac{1}{4} s^{2} M_{Z}^{2}\right) B_{0}\left(M_{Z}^{2}, 0 ; 0\right) \\
& +\left(-\frac{1}{8} \frac{p^{4}}{M_{Z}^{2}}+\frac{5}{2} \frac{p^{2} M_{W}^{2}}{M_{Z}^{2}}+\frac{3}{8} \frac{\left(2 m^{2}\right)^{2}}{M_{Z}^{2}}+\frac{3}{2} \frac{2 m^{2} M_{W}^{2}}{M_{Z}^{2}}+3 \frac{M_{W}^{4}}{M_{Z}^{2}}\right) B_{0}\left(M_{W}^{2}, M_{W}^{2} ; p^{2}\right) \\
& \left.+\left(\frac{1}{8} \frac{p^{4}}{M_{Z}^{2}}+\frac{1}{2} p^{2}+\frac{3}{2} M_{Z}^{2}\right) B_{0}\left(M_{Z}^{2}, M_{Z}^{2} ; p^{2}\right)+\left(\frac{9}{8} \frac{\left(2 m^{2}\right)^{2}}{M_{Z}^{2}}\right) B_{0}\left(2 m^{2}, 2 m^{2} ; p^{2}\right)\right\}, \\
\Sigma_{\underline{\mathcal{W}}}^{T}\left(p^{2}\right)= & \frac{e^{2}}{s^{2} c^{2}}\left\{\left(-\frac{c^{2}}{12} \frac{2 m^{2}-M_{W}^{2}}{p^{2}}+\frac{7}{12} c^{2}\right) A_{0}\left(2 m^{2}\right)\right. \\
& +\left(\frac{c^{2} 2 m^{2}+M_{W}^{2}+6 c^{2} M_{W}^{2}-8 c^{4} M_{W}^{2}}{12 p^{2}}+\frac{2}{3} \frac{c^{2} p^{2}}{M_{W}^{2}}+3 c^{2} \frac{M_{W}^{2}}{2 m^{2}}-\frac{11}{6} c^{2}+\frac{4}{3} c^{4}\right) A_{0}\left(M_{W}^{2}\right) \\
& +\left(\left[-1-7 c^{2}+4 c^{4}+4 c^{6}\right] \frac{M_{W}^{2}}{12 p^{2}}-\frac{2}{3} \frac{c^{4} p^{2}}{M_{Z}^{2}}+\frac{3}{2} \frac{M_{W}^{2}}{2 m^{2}}+\frac{1}{12} c^{2}-\frac{5}{3} c^{4}-\frac{1}{3} c^{6}\right) A_{0}\left(M_{Z}^{2}\right) \\
& +\left(-\frac{2}{3} \frac{c^{2} p^{4}}{M_{W}^{2}}+\frac{5}{3} c^{2} p^{2}+\frac{7}{3} c^{2} M_{W}^{2}\right) B_{0}\left(M_{W}^{2}, M_{W}^{2} ; p^{2}\right)
\end{aligned}
$$




$$
\begin{aligned}
& +\left(\left[-1-6 c^{2}+11 c^{4}-4 c^{8}\right] \frac{M_{W}^{4}}{12 c^{2} p^{2}}+\frac{2}{3} \frac{c^{6} p^{4}}{M_{W}^{2}}+\left[-\frac{1}{12} c^{2}+\frac{14}{3} c^{4}+c^{6}\right] p^{2}\right. \\
& \left.+\left[\frac{5}{6}-\frac{29}{6} c^{2}+\frac{2}{3} c^{4}\right] M_{W}^{2}\right) B_{0}\left(M_{W}^{2}, M_{Z}^{2} ; p^{2}\right) \\
& +\left(-\frac{c^{2}}{12} \frac{\left(2 m^{2}-M_{W}^{2}\right)^{2}}{p^{2}}-\frac{1}{12} c^{2} p^{2}-\frac{1}{6} c^{2} 2 m^{2}+\frac{5}{6} c^{2} M_{W}^{2}\right) B_{0}\left(M_{W}^{2}, 2 m^{2} ; p^{2}\right) \\
& +\left(-\frac{s^{2} c^{4} M_{W}^{4}}{3 p^{2}}+\left(1-c^{4}\right) \frac{2 c^{2} p^{4}}{3 M_{W}^{2}}+\left[\frac{19}{3} c^{2}-\frac{16}{3} c^{4}-c^{6}\right] p^{2}+\left[-\frac{1}{3} c^{2}+\frac{1}{3} c^{4}\right] M_{W}^{2}\right) B_{0}\left(0, M_{W}^{2} ; p^{2}\right) \\
& \left.+\frac{1}{16 \pi^{2}}\left(\left[-\frac{11}{9} c^{2}+\frac{4}{3} c^{4}\right] p^{2}-\frac{1}{6} c^{2} 2 m^{2}+\left[\frac{1}{c^{2}}+2 c^{2}\right] \frac{M_{W}^{4}}{2 m^{2}}+\left[-\frac{1}{6}-\frac{7}{3} c^{2}-\frac{2}{3} c^{4}\right] M_{W}^{2}\right)\right\}, \\
& \Sigma_{\underline{\mathcal{W}}}^{L}\left(p^{2}\right)=\left(\frac{1}{2} \frac{M_{W}^{2}-2 m^{2}}{p^{2}}-\frac{3}{2}\right) A_{0}\left(2 m^{2}\right)+\left(\left[3-4 c^{2}+\frac{1}{2 c^{2}}\right] \frac{M_{W}^{2}}{p^{2}}+\frac{1}{2} \frac{2 m^{2}}{p^{2}}-6 \frac{M_{W}^{2}}{2 m^{2}}-2\right) A_{0}\left(M_{W}^{2}\right) \\
& +\left(\left[-\frac{1}{2}-\frac{7}{2} c^{2}+2 c^{4}+2 c^{6}\right] \frac{M_{Z}^{2}}{p^{2}}+\frac{1}{2}-2 c^{2}+2 c^{4}\right) A_{0}\left(M_{Z}^{2}\right) \\
& +\left(\frac{1}{2} p^{2}-2 M_{W}^{2}\right) B_{0}\left(M_{W}^{2}, M_{W}^{2} ; p^{2}\right) \\
& +\left(\left[-\frac{1}{2 c^{4}}-\frac{3}{c^{2}}+\frac{11}{2}-2 c^{4}\right] \frac{M_{W}^{4}}{p^{2}}+\left[-\frac{1}{2}+2 c^{2}-2 c^{4}\right] p^{2}+\left[-\frac{1}{c^{2}}+3-4 c^{4}\right] M_{W}^{2}\right) B_{0}\left(M_{W}^{2}, M_{Z}^{2} ; p^{2}\right) \\
& +\left(-\frac{1}{2} \frac{\left(2 m^{2}-M_{W}^{2}\right)^{2}}{p^{2}}-2 M_{W}^{2}\right) B_{0}\left(M_{W}^{2}, 2 m^{2} ; p^{2}\right) \\
& +\left(-\frac{2 s^{2} c^{2} M_{W}^{4}}{p^{2}}-2 s^{2} c^{2} p^{2}+\left[-6+2 c^{2}+4 c^{4}\right] M_{W}^{2}\right) B_{0}\left(0, M_{W}^{2} ; p^{2}\right) \\
& +\frac{1}{16 \pi^{2}}\left(\left[-4-\frac{2}{c^{4}}\right] \frac{M_{W}^{4}}{2 m^{2}}+4 s^{2} M_{W}^{2}\right), \\
& \Sigma_{\mathcal{Z}}^{T}\left(p^{2}\right)=\frac{e^{2}}{s^{2} c^{2}}\left\{\left(-\frac{1}{12} \frac{2 m^{2}-M_{Z}^{2}}{p^{2}}+\frac{7}{12}\right) A_{0}\left(2 m^{2}\right)+\left(3 \frac{M_{W}^{2}}{2 m^{2}}+\frac{1}{6}+\frac{4}{3} c^{2}-4 c^{4}\right) A_{0}\left(M_{W}^{2}\right)\right. \\
& +\left(\frac{1}{12} \frac{2 m^{2}-M_{Z}^{2}}{p^{2}}+\frac{3}{2} \frac{M_{Z}^{2}}{2 m^{2}}+\frac{1}{12}\right) A_{0}\left(M_{Z}^{2}\right) \\
& +\left(\left[-\frac{1}{12}+\frac{1}{3} c^{2}+7 c^{4}\right] p^{2}+\left[\frac{5}{3}+\frac{4}{3} c^{2}-4 c^{4}\right] M_{W}^{2}\right) B_{0}\left(M_{W}^{2}, M_{W}^{2} ; p^{2}\right) \\
& +\left(-\frac{1}{12} \frac{\left(2 m^{2}-M_{Z}^{2}\right)^{2}}{p^{2}}-\frac{1}{12} p^{2}-\frac{1}{6} 2 m^{2}+\frac{5}{6} M_{Z}^{2}\right) B_{0}\left(M_{Z}^{2}, 2 m^{2} ; p^{2}\right) \\
& \left.+\frac{1}{16 \pi^{2}}\left(\left[-\frac{1}{9}+\frac{2}{9} c^{2}\right] p^{2}-\frac{1}{6} 2 m^{2}+\left[1+2 c^{4}\right] \frac{M_{Z}^{4}}{2 m^{2}}+\left[-\frac{1}{6}-\frac{1}{3} c^{2}+\frac{4}{3} c^{4}-4 c^{6}\right] M_{Z}^{2}\right)\right\} \\
& \Sigma_{\mathcal{Z}}^{L}\left(p^{2}\right)=\left(-\frac{1}{2} \frac{2 m^{2}-M_{Z}^{2}}{p^{2}}-\frac{3}{2}\right) A_{0}\left(2 m^{2}\right)+\left(-6 \frac{M_{W}^{2}}{2 m^{2}}-2\right) A_{0}\left(M_{W}^{2}\right)+\left(\frac{1}{2} \frac{2 m^{2}-M_{Z}^{2}}{p^{2}}-3 \frac{M_{Z}^{2}}{2 m^{2}}+\frac{1}{2}\right) A_{0}\left(M_{Z}^{2}\right) \\
& +\left(-4 M_{W}^{2}\right) B_{0}\left(M_{W}^{2}, M_{W}^{2} ; p^{2}\right)+\left(\frac{1}{2} p^{2}+2 m^{2}-M_{W}^{2}\right) B_{0}\left(M_{W}^{2}, 2 m^{2} ; p^{2}\right) \\
& +\left(-\frac{1}{2} \frac{\left(2 m^{2}-M_{Z}^{2}\right)^{2}}{p^{2}}-\frac{1}{2} p^{2}-2 m^{2}-M_{Z}^{2}\right) B_{0}\left(M_{Z}^{2}, 2 m^{2} ; p^{2}\right)+\frac{1}{16 \pi^{2}}\left(-4 \frac{M_{W}^{4}}{2 m^{2}}-2 \frac{M_{Z}^{4}}{2 m^{2}}\right), \\
& \Sigma_{\mathcal{Z} \mathcal{A}}^{T}\left(p^{2}\right)=\frac{e^{2}}{s c}\left\{\left(-\frac{2}{3}+4 c^{2}\right) A_{0}\left(M_{W}^{2}\right)+\left(\left[-\frac{2}{3}+4 c^{2}\right] M_{W}^{2}+\left[-\frac{1}{6}-7 c^{2}\right] p^{2}\right) B_{0}\left(M_{W}^{2}, M_{W}^{2} ; p^{2}\right)\right.
\end{aligned}
$$




$$
\begin{aligned}
& \left.+\frac{1}{16 \pi^{2}}\left(-\frac{2}{3} M_{W}^{2}+4 c^{2} M_{W}^{2}-\frac{1}{9} p^{2}\right)\right\} \\
\Sigma_{\mathcal{A Z}}^{T}\left(p^{2}\right)= & \Sigma_{\mathcal{Z} \mathcal{A}}^{T}\left(p^{2}\right) \\
\Sigma_{\mathcal{Z} \mathcal{A}}^{L}\left(p^{2}\right) \equiv & 0 \\
\Sigma_{\mathcal{A}}^{T}\left(p^{2}\right)= & -e^{2}\left\{4 A_{0}\left(M_{W}^{2}\right)+\left(4 M_{W}^{2}-7 p^{2}\right) B_{0}\left(M_{W}^{2}, M_{W}^{2} ; p^{2}\right)+\frac{1}{16 \pi^{2}} 4 M_{W}^{2}\right\} \\
\Sigma_{\mathcal{A}}^{L}\left(p^{2}\right) \equiv & 0 .
\end{aligned}
$$

The tadpole contribution $A_{0}$ and the two-point integral $B_{0}$ which appear in Eqs. $(\overline{\mathrm{C} 1})-(\overline{\mathrm{C} 10})$ are defined by the following equations:

$$
\begin{aligned}
A_{0}\left(M^{2}\right) & =\int \frac{d^{d} q}{(2 \pi)^{d}} \frac{1}{\left(q^{2}+M^{2}\right)}, \\
B_{0}\left(M_{1}^{2}, M_{2}^{2} ; p^{2}\right) & =\int \frac{d^{d} q}{(2 \pi)^{d}} \frac{1}{\left(q^{2}+M_{1}^{2}\right)} \frac{1}{\left((q+p)^{2}+M_{2}^{2}\right)} .
\end{aligned}
$$

Note that we are working in Euclidean space-time.

[1] L. D. Faddeev and V. N. Popov, Phys. Lett. 25B, 29 (1967).

[2] C. Becchi, A. Rouet, and R. Stora, Commun. Math. Phys. 42, 127 (1975); Ann. Phys. (N.Y.) 98, 287 (1976).

[3] W. Beenakker et al., "Reports of the Working Group on Physics at LEP2", CERN Yellow Report No. CERN 96-01, edited by G. Altarelli, T. Sjöstrand, and F. Zwirner, hep-ph/9602351; G. Montagna, O. Nicrosini, and F. Piccinini, Riv. Nuovo Cimento 21, 1 (1998); W. Beenakker and A. Denner, Acta Phys. Pol. B 29, 2821 (1998).

[4] M. E. Peskin and T. Takeuchi, Phys. Rev. Lett. 65, 964 (1990); Phys. Rev. D 46, 381 (1992).

[5] P.F. Kelly, R. Kobes, and G. Kunstatter, Phys. Rev. D 50, 7592 (1994) and references therein.

[6] J. Bijnens and J. Prades, Nucl. Phys. B490, 239 (1997); B. Moussallam, ibid. B504, 381 (1997); M. Knecht and R. Urech, ibid. B519, 329 (1998).

[7] R. Dashen, Phys. Rev. 183, 1245 (1969); G. Ecker, J. Gasser, A. Pich, and E. de Rafael, Nucl. Phys. B321, 311 (1989); R. Urech, ibid. B433, 234 (1995); H. Neufeld and H. Rupertsberger, Z. Phys. C 71, 131 (1996).

[8] S. Weinberg, Physica A 96, 327 (1979); J. Gasser and H. Leutwyler, Ann. Phys. (N.Y.) 158, 142 (1984); Nucl. Phys. B250, 465 (1985).

[9] D. Espriu and J. Matias, Phys. Lett. B 341, 332 (1995).

[10] A. Nyffeler and A. Schenk, Phys. Rev. D 53, 1494 (1996).

[11] W. Beenakker et al., Nucl. Phys. B500, 255 (1997).

[12] J.M. Cornwall, in Proceedings of the French-American Seminar on Theoretical Aspects of Quantum Chromodynamics, edited by J.W. Dash, Centre de Physique Théorique, Marseille, 1982, Report No. CPT-81/P-1345; Phys. Rev. D 26, 1453 (1982); J.M. Cornwall and J. Papavassiliou, ibid. 40, 3474 (1989); J. Papavassiliou, ibid. 41, 3179 (1990); G. Degrassi and A. Sirlin, ibid. 46, 3104 (1992).

[13] For a review on more recent developments within the pinch technique framework, see J. Papavassiliou, in the "Proceedings of the 6th Hellenic School and Workshop on Elementary Particle Physics", Corfu, Greece, 1998, hep-ph/9905328.

[14] G. Degrassi, B.A. Kniehl, and A. Sirlin, Phys. Rev. D 48, 3963 (1993).

[15] L.F. Abbott, Nucl. Phys. B185, 189 (1981); Acta Phys. Pol. B 13, 33 (1982) and references therein.

[16] A. Denner, G. Weiglein, and S. Dittmaier, Phys. Lett. B 333, 420 (1994); Nucl. Phys. B440, 95 (1995); A. Denner, S. Dittmaier, and G. Weiglein, Acta Phys. Pol. B 27, 3645 (1996).

[17] A. Schenk, Karlsruhe University Report No. TTP-96-47, 1996, hep-ph/9610279.

[18] A. Nyffeler and A. Schenk, Ann. Phys. (N.Y.) 241, 301 (1995).

[19] A. Nyffeler and A. Schenk, DESY Report No. DESY-99-088, 1999, hep-ph/9907294.

[20] T. Appelquist and C. Bernard, Phys. Rev. D 22, 200 (1980); T. Appelquist, in Gauge Theories and Experiments at High Energies, edited by K. C. Bowler and D. G. Sutherland (Scottish University Summer School in Physics, St. Andrews, 1980); A. C. Longhitano, Phys. Rev. D 22, 1166 (1980); Nucl. Phys. B188, 118 (1981); T. Appelquist and G.-H. Wu, Phys. Rev. D 48, 3235 (1993); 51, 240 (1995).

[21] O. Steinmann, Ann. Phys. (N.Y.) 157, 232 (1984) and references therein. 
[22] For a recent review, see R. Horan, M. Lavelle, and D. McMullan, Pramana, J. Phys. 51, 317 (1998), hep-th/9810089, and references therein.

[23] G. A. Vilkovisky, Nucl. Phys. B234, 125 (1984); B. S. DeWitt, in Quantum Field Theory and Quantum Statistics: Essays in Honour of the Sixtieth Birthday of E. S. Fradkin, edited by I. A. Batalin, C. J. Isham, and G. A. Vilkovisky (Adam Hilger, Bristol, 1987).

[24] I. Montvay and G. Münster, Quantum Fields on a Lattice, Cambridge Monographs on Mathematical Physics (Cambridge University Press, Cambridge, England, 1997).

[25] T. Banks and E. Rabinovici, Nucl. Phys. B160, 349 (1979).

[26] G. 't Hooft, in Recent Developments in Gauge Theories, Cargèse, France, 1979, edited by G. 't Hooft et al. (Plenum Press, New York, 1980), Lecture II, p. 117; for a recent account, see G. 't Hooft, in International School of Nuclear Physics, 20th Course: Heavy Ion Collisions from Nuclear to Quark Matter, Erice, Italy, 1998, edited by A. Faessler (Pergamon Press, New York, 1999) [Prog. Part. Nucl. Phys. (in press)], hep-th/9812204.

[27] F. Jegerlehner and J. Fleischer, Phys. Lett. 151B, 65 (1985); Acta Phys. Pol. B 17, 709 (1986) and references therein; M. Chanowitz, M. Golden, and H. Georgi, Phys. Rev. D 36, 1490 (1987).

[28] R. Haag, Phys. Rev. 112, 669 (1958).

[29] A. M. Polyakov, Nucl. Phys. B120, 429 (1977); Phys. Lett. 103B, 207 (1981).

[30] J. Schwinger, Phys. Rev. 82, 664 (1951); P. B. Gilkey, The Index Theorem and the Heat Equation (Publish or Perish, Boston, 1974).

[31] For a calculation in a general $R_{\xi}$-gauge, see J. Fleischer and F. Jegerlehner, Phys. Rev. D 23, 2001 (1981).

[32] M. Lavelle and D. McMullan, Phys. Rep. 279, 1 (1997).

[33] K. Haller and L. Chen, talk delivered at the XXIXth International Conference on High Energy Physics, Vancouver, Canada, 1998, hep-th/9808044. 